\title{
A Local Separation Principle via Dynamic Approximate Feedback and Observer Linearization for a Class of Nonlinear Systems
}

\author{
M. Sassano and A. Astolfi, Fellow, IEEE
}

\begin{abstract}
A separation principle for a class of nonlinear systems inspired by the techniques of feedback linearization and observer design with linear error dynamics is discussed. The output feedback construction combines strategies for approximate feedback linearization and observer design, which are of interest per se, yielding a dynamic control law that ensures a linear, spectrally assignable, behavior from the certainty equivalence input mismatch to the extended state of the system and the observer. The first ingredient, namely the approximate feedback linearization strategy, can be applied, under mild conditions, also to nonlinear systems that are linearly uncontrollable - or that do not possess a well-defined relative degree in the case of a given output function - yet providing a chain of integrators of length equal to the dimension of the state in the transformed coordinates. Interestingly, a systematically designed nonlinear inner loop enables use of linear design techniques, e.g. pole placement. The observer design, on the other hand, employs an additional dynamic extension that allows to assign the local dynamic behavior of the error dynamics independently from its zeros, differently from the classic high-gain observer design. The paper is concluded by presenting several numerical simulations, including an output tracking control problem for the Ball and Beam model that does not possess a well-defined relative degree.
\end{abstract}

Index Terms-Feedback linearization, Nonlinear systems, Observers design, Stability of NL systems

\section{INTRODUCTION}

The problem of exact feedback linearization consists in determining a state feedback control law and a change of coordinates in the state-space with the objective of transforming a given nonlinear control system into a linear and controllable one [13]. It has been shown that the solution to the above problem hinges upon the existence of an output function such that the system possesses a well-defined relative degree equal to the dimension of the state of the original nonlinear system [13]. The construction of such a function can be recast into the

M. Sassano is with the Dipartimento di Ingegneria Civile e Ingegneria Informatica, Università di Roma "Tor Vergata", Via del Politecnico, 1 00133 Roma, Italy (Email: mario.sassano@uniroma2.it).

A. Astolfi is with the Department of Electrical and Electronic Engineering, Imperial College London, London SW7 2AZ, UK and with the Dipartimento di Ingegneria Civile e Ingegneria Informatica, Università di Roma "Tor Vergata", Via del Politecnico, 100133 Roma, Italy (Email: a.astolfi@ic.ac.uk).

This work has been partially supported by the European Union's Horizon 2020 Research and Innovation Programme under grant agreement No 739551 (KIOS CoE).
- typically daunting - task of solving a system of first-order linear partial differential equation.

As a consequence, in the last decades significant research effort has been devoted to the construction of approximate solutions to the problem of exact feedback linearization [3], [4], [23]. In the comprehensive survey [9] the different approaches are discussed and categorized depending on the specific notion of approximation considered, namely partial linearization techniques, initiated in the seminal paper [23], linearizationoriented modeling [8], [10], nonlinearity measures [7], [30] and linear model-matching. In [12], [15], [16], [25] state feedbacks are designed in such a way that the nonlinear system, though not feedback linearized, exhibits a linear inputoutput behavior. In particular, the structure of the closed-loop systems obtained in [15] is reminiscent of the one derived here. However, two main differences must be stressed. First, the dimension of the linear subsystem matches that of the original nonlinear system and, second, the construction is not based on the definition of specific controlled-invariant distributions but relies on a systematic design technique.

Similarly to the above state feedback stabilization problem, also for the observer design task several solutions have been proposed in the literature. Most of such implementations are intrinsically related to the well-known high-gain observer design [21], [22], [33] or to Luenberger-like observers [1], [19], [24]. A somewhat different direction of research has been created with the definition of the Observer Linearization Problem [13], in which the objective consists in constructing observers that yield a linear and spectrally assignable error dynamics, possibly modulo a change of coordinates. The construction, however, is based on rather strong assumptions and involves the solution of a partial differential equation.

It is then not surprising that, in its most desirable configuration the solution of the output feedback stabilization problem - which is of paramount importance from theoretical and practical perspectives, as testified by the extremely large literature on the topic - typically hinges upon the combination of the two separate ingredients above: the construction of a stabilizing state feedback and the design of an observer to reconstruct the state of the system, [6], [14], [17], [20], [26]-[28], [31], [32]. It is well-known, as entailed by the celebrated Separation Principle, that for linear systems these two components may be designed independently from each other and then combined to create an output feedback stabilizer [18], whereas a similar implementation in the nonlinear setting 
has represented a long-standing challenge.

The main contribution of this paper is threefold. First, we discuss a control design methodology that allows to immerse any nonlinear system with state of dimension $n$ into an extended system that comprises a chain of $n$ integrators, hence defining an approximate feedback linearization strategy. The result is achieved under mild assumptions on the nonlinear system, which is not required for instance to be linearly controllable, a necessary condition (also sufficient for planar nonlinear systems) for exact feedback linearization. The construction does not involve the solution of any pde, while exploiting the evolution of a dynamic extension driven by the state of the system. The overall architecture consists of a nonlinear inner loop that enables use of linear design techniques, e.g. pole placement, even for linearly uncontrollable systems. The technique is also employed to solve two classical design problems for nonlinear control systems: local asymptotic stabilization of a desired equilibrium point and (local) asymptotic tracking of reference signals, even for systems not possessing a well-defined relative degree. Then, inspired by the spirit of the observer linearization problem and mimicking (in a dualized manner) the rationale behind the construction of the feedback linearizing control law, we propose an observer design technique that allows to arbitrarily assign the poles of the local behavior of the error dynamics without affecting (part of) its zeros. Finally, the two above techniques are combined together to yield an output feedback design architecture such that the behavior from the certainty equivalence input mismatch to the extended state of the system and the observer is linear and spectrally assignable.

The rest of the paper is organized as follows. In Section II-A the main results concerning approximate feedback linearization are discussed in the case of planar systems. Such results consist essentially in illustrating the design of dynamic control laws that allow, firstly, to obtain an extended system comprising a double integrator, namely characterized by a linear input/output behavior, in which the trajectories of the original system are immersed, then to enforce (local) asymptotic stability of a desired equilibrium point and finally to achieve asymptotic tracking of a reference signal. Similar statements are then extended to general nonlinear systems in Section II-B. The main topic of Section III is the design of observers inspired by the approach of observer linearization for autonomous nonlinear systems, hence providing a dual implementation with respect to the topics of Sections II-A and II-B. The two above techniques are then combined to define an output feedback design strategy that enjoys the interesting features of each of the approaches as extensively discussed in Section IV. Finally, the performances of the proposed control design techniques are assessed, in Section $\mathrm{V}$, by means of several numerical examples, encompassing a locally uncontrollable planar system with a non-hyperbolic equilibrium point and the Ball and Beam model, while conclusions are drawn in Section VI. A preliminary version of this paper has appeared in [29]. With respect to [29], herein we present novel results on observer design and a nonlinear separation principle, in addition to the proofs of the main results for approximate feedback linearization as well as more detailed discussions.

\section{Approximate FeEdback Linearization for NONLINEAR SYSTEMS}

The aim of this section is to present a systematic technique that allows, under mild assumptions, to immerse a nonlinear system of dimension $n$ into an extended system that comprises a chain of $n$ integrators - namely providing a linear input/output behavior - which contain all the components of the original nonlinear system. The result is achieved by means of a coordinate transformation and a dynamic feedback, and does not rely on feedback linearizability. The approach is initially discussed in the case of planar nonlinear systems to highlight the rationale of the construction without the additional burden of notation yielded by higher-dimensional systems, which are dealt with in Section II-B. Note that a similar exposition approach is adopted also in Sections III and IV.

\section{A. Planar Systems}

Consider planar nonlinear systems described by

$$
\dot{x}=f(x)+g(x) u,
$$

with $x(t)=\left[x_{1}(t), x_{2}(t)\right]^{\top} \in \mathbb{R}^{2}$ denoting the state of the system and $u(t) \in \mathbb{R}$ denoting the control input. The mappings $f: \mathbb{R}^{2} \rightarrow \mathbb{R}^{2}$ and $g: \mathbb{R}^{2} \rightarrow \mathbb{R}^{2}$ are assumed to be sufficiently smooth. Moreover, suppose that $x_{e} \in \mathbb{R}^{2}$ is an equilibrium point of system (1) with $u=0$, namely $f\left(x_{e}\right)=0$. Throughout the rest of the paper we suppose that $x_{e}=0$. The objective of the following statement consists in extending the machinery associated to feedback linearization also to systems that are not feedback linearizable in the classical sense. In fact, note that the planar system (1) is locally feedback linearizable if and only if its linear approximation around $x_{e}$ is controllable, which is not assumed here. To provide a concise statement, let $\left.A \triangleq \nabla_{x} f(x)\right|_{x=0}$ and $B \triangleq g(0)$ denote the matrices defining the linear approximation of (1) around the origin. Moreover, the Lie derivative of the function $h$ along the vector field $f$, namely $(\partial h / \partial x) f(x)$, is denoted by $L_{f} h$. To avoid cumbersome notation, recall the standard notation for repeated Lie derivatives, namely

$$
\begin{aligned}
L_{f_{1}} h(x) & =\frac{\partial h(x)}{\partial x} f_{1}(x), \\
L_{f_{2}} L_{f_{1}} h(x) & =\frac{\partial L_{f_{1}} h(x)}{\partial x} f_{2}(x),
\end{aligned}
$$

with function $h: \mathbb{R}^{n} \rightarrow \mathbb{R}$ and mappings $f_{i}: \mathbb{R}^{n} \rightarrow \mathbb{R}^{n}$ for $i=1,2$, or, if $f_{1}=f_{2}=f, L_{f}^{2} h(x)=\left(\partial L_{f} h(x) / \partial x\right) f(x)$.

Proposition 1: Consider the planar nonlinear system (1) and define the mapping $\psi: \mathbb{R}^{3} \rightarrow \mathbb{R}^{3}$ as

$$
\left[\begin{array}{l}
z \\
\xi
\end{array}\right]=\left[\begin{array}{c}
\psi_{z}(x, \xi) \\
\xi
\end{array}\right]=\left[\begin{array}{c}
H x+\xi \\
L_{f}(H x)-\kappa \xi \\
\xi
\end{array}\right] \triangleq \psi(x, \xi) .
$$

Suppose that there exist a matrix $H \in \mathbb{R}^{1 \times 2}$ and a constant $\kappa \in \mathbb{R}$ such that (C1)

1) the matrix

$$
\mathcal{O}=\left[\begin{array}{ll}
H^{\top} & (H A)^{\top}
\end{array}\right]^{\top}
$$

has rank 2; 


$$
\text { 2) } H A B+\kappa H B \neq 0 \text {. }
$$

Consider the system (1) in closed loop with

$$
\left\{\begin{aligned}
u & =\frac{1}{r(x)}(s(x, \xi)+v) \\
\dot{\xi} & =-\kappa \xi-L_{g}(H x) u
\end{aligned}\right.
$$

with $r(x)=L_{g} L_{f}(H x)+\kappa L_{g}(H x)$ and $s(x, \xi)=$ $-L_{f}^{2}(H x)-\kappa^{2} \xi$. Then, the behavior from the input $v$ to the output $\hat{y} \triangleq z_{1}$ of system (1)-(5) in the $(z, \xi)$-coordinates is locally described by a double integrator, namely $\hat{y}^{(2)}=v$. Moreover, the Jacobian matrix $\left.\nabla_{x} \psi_{z}(x, \xi)\right|_{(x, \xi)=(0,0)}$ is nonsingular.

Proof: To begin with note that condition $(C 1)$ implies that the mapping $\psi$ defined in (3) is a local diffeomorphism around $(x, \xi)=(0,0)$, hence a valid local change of coordinates. By employing the definition of the control input $u$, which is locally well-defined by item $(C 2)$ of the statement since $r(0)=H A B+\kappa H B$, and the dynamics of $\xi$ as in (5), in the transformed coordinates the system (1)-(5) is described by

$$
\begin{aligned}
\dot{z}_{1} & =z_{2} \\
\dot{z}_{2} & =v \\
\dot{\xi} & =(-\kappa \xi-H g(x)(\alpha(x, \xi)+\beta(x) v))
\end{aligned}
$$

with $\alpha(x, \xi)=s(x, \xi) r(x)^{-1}$ and $\beta(x)=r(x)^{-1}$. Note that the $z$-subsystem of (6) is independent of $\xi$. Finally, noting that $\left.\nabla_{x} \psi_{z}(x, \xi)\right|_{(x, \xi)=(0,0)}=\mathcal{O}$, the second claim follows immediately from condition $(C 1)$.

Remark 1: The property that the Jacobian matrix $\nabla_{x} \psi_{z}(x, \xi)$ evaluated at $(x, \xi)=(0,0)$ is non-singular implies that all the components of the state of system (1) are included in the double integrator. As a consequence, and as shown in the following, convergence of $(z(t), \xi(t))$ to the origin, in the transformed coordinates, implies local convergence of $x(t)$ to zero in the original coordinates.

Remark 2: The conditions $(C 1)$ and $(C 2)$ on the matrix $H$ are natural: the first-order approximation of system (1) around the origin should be observable via the user selected output $y=H x$ and the corresponding transfer function of the linear approximation between $u$ and the same output $y$ is not identically equal to zero, respectively. As additional insight on $(C 1)$, note that it is possible to determine a row vector $H$ such that the pair $(H, A)$ is observable if and only if the matrix $A$ does not possess more than one Jordan block associated to the same eigenvalue, which is in turn a generically verified property, namely it holds with probability one for a random selection of the entries in $A$. Moreover, it is not required that the first-order approximation of (1) be controllable, i.e. (1) may not be feedback linearizable [13].

To put the above results into perspective note that, for a given output function $h$, the conditions $(C 1)$ and $(C 2)$ of Proposition 1 may hold even if the system does not possess a well-defined relative degree around the origin, as pointed out in the following example (see also the example on the Ball and Beam model in Section V-C).
Example. Consider the nonlinear system described by

$$
\begin{aligned}
\dot{x}_{1} & =x_{1}+x_{2}+x_{1} u \\
\dot{x}_{2} & =x_{1} x_{2}+u \\
y & =x_{1}
\end{aligned}
$$

Since $L_{g} h(x)=x_{1}$ vanishes for $x=0$, but it is not identically equal to zero in any neighborhood of the origin, the system (7) does not possess a well-defined relative degree at the origin. Moreover, as it is well-known, see e.g. [13], such property cannot be enforced even by resorting to a construction similar to those employed in [34], namely by defining $u=\zeta, \dot{\zeta}=v$ and considering the relative degree between $y$ and $v$. Nonetheless, it can be easily checked that condition $(C 1)$ holds and $H A B=1$ with $H=\left[\begin{array}{ll}1 & 0\end{array}\right]$, i.e. condition $(C 2)$ can be satisfied, hence one can apply the construction of Proposition 1 and obtain a well-defined chain of (two) integrators. The result is achieved essentially by replacing the choice $\dot{\zeta}=v$ with $\dot{\xi}=-\kappa \xi-x_{1} u$, which yields the system

$$
\begin{aligned}
\dot{z}_{1}= & z_{2}, \quad \dot{z}_{2}=v \\
\dot{\xi}= & -\kappa \xi-\frac{z_{1}-\xi}{1+(\kappa+1)\left(z_{1}-\xi\right)}\left(-z_{2}-\left(\kappa+\kappa^{2}\right) \xi\right. \\
& \left.-\left(z_{1}-\xi\right)\left(z_{2}-z_{1}+(\kappa+1) \xi\right)+v\right) .
\end{aligned}
$$

As anticipated above, such chain of integrators is characterized by the property that local convergence of $(z(t), \xi(t))$ in (8) to zero implies a similar conclusion for $x(t)$ in (7).

By exploiting the nonlinear construction in (5), a linear control design methodology can be now employed, with additional assumptions discussed below, to stabilize the zero equilibrium of (1) or enforce asymptotic tracking of a desired reference signal, as detailed in the following statements. Note that the condition below is only sufficient for asymptotic stability of the zero equilibrium of the $\xi$ dynamics, while alternative nonlinear techniques can be employed for the analysis, should the following condition fail.

Proposition 2: Consider the planar nonlinear system (1). Suppose that there exist a matrix $H \in \mathbb{R}^{1 \times 2}$ and a constant $\kappa \in \mathbb{R}$ such that conditions $(C 1)$ and $(C 2)$ hold. Suppose in addition that (C3)

1) the constant

$$
\varrho_{\kappa} \triangleq-\kappa-\nabla_{\xi}\left[\left.H g(x) \alpha(x, \xi)\right|_{(x, \xi)=\psi^{-1}(z, \xi)}\right]_{(z, \xi)=(0,0)}
$$

is negative.

Consider the system (1) in closed loop with (5), with

$$
v=-c_{1}(H x+\xi)-c_{2}\left(L_{f}(H x)-\kappa \xi\right) .
$$

Then there exists a neighborhood $\mathcal{U}$ of $z_{o}=\psi_{z}(0,0)=0$ such that for any $\lambda \in \mathbb{R}_{+}$there exist $c_{1}>0$ and $c_{2}>0$ such that $\|z(t)\|<e^{-\lambda t}\|z(0)\|$ for all $z(0) \in \mathcal{U}$ and moreover the equilibrium point $(x, \xi)=(0,0)$ of (1)-(5)-(10) is locally asymptotically stable.

Proof: The proof of the claim follows steps identical to those in the proof of Proposition 1 in showing that the interconnected system (1)-(5) is described by (6), while the choice of $v$ as in 
(10), namely $v=-c_{1} z_{1}-c_{2} z_{2}$, is such that the corresponding trajectories of the $z$-subsystem exponentially converge to the origin. The rate of convergence can be arbitrarily assigned by selecting the positive constants $c_{1}$ and $c_{2}$. Note now that condition $(C 3)$ implies asymptotic stability of the first-order approximation of the (unforced, i.e. with $z=0$ ) $\xi$-subsystem, hence local input-to-state stability of the $\xi$-subsystem with respect to $z$. Therefore, local asymptotic stability of the zero equilibrium in the $(z, \xi)$-coordinates of (6)-(10) can be concluded. Thus, $\lim _{t \rightarrow \infty} x(t)=0$ by the structure of the change of coordinates $\psi$ defined in (3).

Despite the fact the proof of stability above is carried out by employing linearization arguments, it is interesting to point out that the approach yields a systematic nonlinear design strategy, consisting of an inner nonlinear feedback, that subsequently enables the use of linear tools, e.g. pole placement, also for nonlinear systems that are not linearly controllable.

Example. Consider the planar nonlinear system (7) and the transformed system (8). It can be shown that $\varrho_{\kappa}=-\kappa$, hence condition $(C 3)$ of Proposition 2 holds for any $\kappa>0$ and the zero equilibrium of the extended system (8) can be rendered locally asymptotically stable by a linear feedback of the form $v=K z$, with $K=\left[k_{1} k_{2}\right]$, for any $k_{1}<0$ and $k_{2}<0$.

The results of Proposition 2 entail that a desired (linear) output of the system $y=H x$ is forced to converge exponentially fast to an exogenous signal that asymptotically tends to zero. Moreover, the above construction is achieved by avoiding the solution of any pde, hence by means of a systematic and constructive procedure. This aspect is illustrated in the numerical example of Section V-A. The above discussion motivates the following result in which the construction of Proposition 2 is employed to enforce approximate tracking of a desired signal. Let $y_{d}$ denote a desired output trajectory and $\dot{y}_{d}, \ddot{y}_{d}$ its first and second order time derivatives, respectively, assumed well-defined. The following assumption characterizes the set of admissible reference trajectories.

Assumption 1: The signal $y_{d}$ is admissible if $y_{d}, \dot{y}_{d}, \ddot{y}_{d}$ are continuous for all $t \geq 0$ and bounded.

To provide a concise statement consider the control law

$$
\begin{aligned}
v & =-c_{1}\left(H x+\xi-y_{d}\right)-c_{2}\left(L_{f}(H x)-\kappa \xi-\dot{y}_{d}\right)+\ddot{y}_{d} \\
& \triangleq \sigma_{p}(x, \xi, t)
\end{aligned}
$$

with $c_{1}>0$ and $c_{2}>0$ and let the mapping $\eta_{p}(x, \xi, t)=$ $-\kappa \xi-H g(x)\left(\alpha(x, \xi)+\beta(x) \sigma_{p}(x, \xi, t)\right)$ denote the closedloop dynamics of the $\xi$-subsystem in (6) and $\bar{\eta}_{p}(z, \xi, t)=$ $\left.\eta_{p}(x, \xi, t)\right|_{(x, \xi)=\psi^{-1}(z, \xi)}$ the corresponding description in the $(z, \xi)$-coordinates. Moreover, let $y_{R} \triangleq\left(y_{d}, \dot{y}_{d}\right)$ be a compact notation for $y_{d}$ and its time derivative.

Proposition 3: Consider the planar nonlinear system (1) together with a given output $y=C x$. Suppose that there exists a constant $\kappa \in \mathbb{R}$ such that $C$ and $\kappa$ satisfy conditions $(C 1)$ and $(C 2)$, with $C$ in place of $H$. Consider system (1) in closed loop with (5), with $v$ selected as in (11). Suppose that the solution $\xi_{s s}(t)$ of

$$
\dot{\xi}(t)=\bar{\eta}_{p}\left(y_{R}(t), \xi(t), t\right),
$$

with $\xi_{s s}(0)=0$, is defined for all $t \geq 0$, bounded and uniformly asymptotically stable ${ }^{1}$. Then for any $\varepsilon>0$ there exists $\delta>0$ such that $\left\|z_{i}(0)-y_{d}^{(i-1)}(0)\right\|<\delta, i=1,2$, and $\|\xi(0)\|<\delta$ imply that $\left\|z_{1}(t)-y_{d}(t)\right\|<\varepsilon$ and

$$
\left\|y(t)-y_{d}(t)\right\| \leq \varepsilon+\left\|\xi_{s s}(t)\right\|,
$$

for all $t \geq 0$ and for any sufficiently small $y_{d}^{(i)}(t)$.

Proof: To begin with note that by assumptions $(C 1)$ and $(C 2)$ the change of coordinates (3) and the control law (5), respectively, are well-defined, locally around the origin, and in the $(z, \xi)$-coordinates the system is described by the equations (6) with $v$ as in (11). Moreover, considering the tracking error $e(t)=z(t)-y_{R}(t)$, system (6)-(11) can be rewritten as

$$
\begin{aligned}
& \dot{e}(t)=K e(t) \\
& \dot{\xi}(t)=\bar{\eta}_{p}\left(e(t)+y_{R}(t), \xi(t), t\right),
\end{aligned}
$$

with $K$ a lower companion matrix, the characteristic polynomial of which is $s^{2}+c_{2} s+c_{1}$, hence Hurwitz since $c_{1}>0$, $c_{2}>0$. Introducing now the mismatch $w(t)=\xi(t)-\xi_{s s}(t)$, system (14) can be described by the equations

$$
\begin{aligned}
\dot{e}(t)= & K e(t), \\
\dot{w}(t)= & \bar{\eta}_{p}\left(e(t)+y_{R}(t), w(t)+\xi_{s s}(t), t\right) \\
& -\bar{\eta}_{p}\left(y_{R}(t), \xi_{s s}(t), t\right) \triangleq F(e(t), w(t), t) .
\end{aligned}
$$

The function $F(e, w, t)$ is locally Lipschitz in $(e, w)$, by smoothness of $\bar{\eta}_{p}$, uniformly in $t$, namely the Lipschitz constant does not depend on time, by boundedness of $y_{R}(t)$, $\ddot{y}_{d}(t)$ and $\xi_{s s}(t)$, and the zero equilibrium of $\dot{w}=F(0, w, t)$ is uniformly asymptotically stable, by uniform asymptotic stability of the solution $\xi_{s s}(t)$ of (12). Then, the results of [13, Appendix B2] can be applied, showing that $\left(0, \xi_{s s}(t)\right)$ is a uniformly stable solution of (14), i.e. for any $\varepsilon>0$, there exists $\delta>0$ such that $\left\|z_{i}(0)-y_{d}^{(i-1)}(0)\right\|<\delta$, $i=1,2,\|\xi(0)\|<\delta$ imply that $\left\|z_{i}(t)-y_{d}^{(i-1)}(t)\right\|<\varepsilon / 2$ and $\left\|\xi(t)-\xi_{s s}(t)\right\|<\varepsilon / 2$, for all $t \geq 0$. Finally, note that

$$
\begin{aligned}
& \left\|y(t)-y_{d}(t)\right\|=\left\|z_{1}(t)-\xi(t)+\xi_{s s}(t)-\xi_{s s}(t)-y_{d}(t)\right\| \\
& \leq\left\|z_{1}(t)-y_{d}(t)\right\|+\left\|\xi(t)-\xi_{s s}(t)\right\|+\left\|\xi_{s s}(t)\right\| \\
& \leq \varepsilon / 2+\varepsilon / 2+\left\|\xi_{s s}(t)\right\|=\varepsilon+\left\|\xi_{s s}(t)\right\|
\end{aligned}
$$

proving the claim.

The results of Proposition 3 show that the (asymptotic) tracking error is bounded and its bound may be explicitly, and $a$ priori, quantified by means of the steady-state response of the system (5). Moreover, note that the term $\left\|\xi_{s s}(t)\right\|$ in the bound becomes arbitrarily small in the presence of reference trajectories such that $y_{d}(t)$ and its time derivatives are arbitrarily small. Interestingly, the result can be employed to solve tracking problems even for nonlinear systems that do not possess a well-defined relative degree at the origin.

\footnotetext{
${ }^{1}$ In the case of constant reference signals $y_{d}$, the condition on the motion $\xi_{s s}(t)$ can be equivalently reformulated in terms of local asymptotic stability properties of the zero equilibrium in $\xi$ for the vector field $\bar{\eta}_{p}(0, \xi)$.
} 


\section{B. General Nonlinear Systems}

The extension of the input/output linearization methodology, introduced in the previous section for planar systems, to general nonlinear systems is the topic of this section. Towards this end consider nonlinear systems described by

$$
\dot{x}=f(x)+g(x) u,
$$

with $x(t) \in \mathbb{R}^{n}$ and $u(t) \in \mathbb{R}$. Note that the proofs of the following results can be obtained by straightforward adaptation of the proof of the analogous results in Section II-A.

Proposition 4: Consider the nonlinear system (16) and define the mapping $\psi: \mathbb{R}^{2 n-1} \rightarrow \mathbb{R}^{2 n-1}$ as

$$
\left[\begin{array}{c}
z \\
\xi
\end{array}\right]=\psi(x, \xi)=\left[\begin{array}{c}
H x+\xi_{1} \\
L_{f}(H x)+\xi_{2} \\
L_{f}^{2}(H x)+\xi_{3} \\
\vdots \\
L_{f}^{n-1}(H x)-\sum_{j=1}^{n-1} \kappa_{j} \xi_{j} \\
\xi
\end{array}\right]
$$

with $\xi \in \mathbb{R}^{n-1}$. Suppose that there exist a matrix $H \in \mathbb{R}^{1 \times n}$ and constants $\kappa_{j} \in \mathbb{R}, j=1, \ldots, n-1$, such that (C1')

1) the matrix

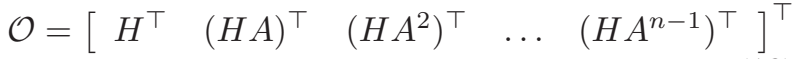

has rank $\mathrm{n}$;

2) $H A^{n-1} B+\sum_{j=1}^{n-1} \kappa_{j} H A^{j-1} B \neq 0$.

Consider the system (16) in closed loop with

$$
\left\{\begin{aligned}
u & =\frac{-L_{f}^{n}(H x)+\sum_{i=1}^{n-1} \kappa_{i} \kappa_{n-1} \xi_{i}-\sum_{j=1}^{n-2} \kappa_{j} \xi_{j+1}+v}{L_{g} L_{f}^{n-1}(H x)+\sum_{j=1}^{n-1} \kappa_{j} L_{g} L_{f}^{j-1}(H x)} \\
\dot{\xi}_{1} & =\xi_{2}-L_{g}(H x) u, \\
\dot{\xi}_{2}= & \xi_{3}-L_{g} L_{f}(H x) u, \\
\vdots & \\
\dot{\xi}_{n-1}= & -\sum_{i=1}^{n-1} \kappa_{i} \xi_{i}-L_{g} L_{f}^{n-2}(H x) u .
\end{aligned}\right.
$$

Then, the behavior from the input $v$ to the output $\hat{y} \triangleq z_{1}$ of system (16)-(19) in the $(z, \xi)$-coordinates is locally described by a chain of $n$ integrators, namely $\hat{y}^{(n)}=v$. Moreover, the Jacobian matrix $\left.\nabla_{x} \psi_{z}(x, \xi)\right|_{(x, \xi)=(0,0)}$ is non-singular.

Remark 3: By arguments identical to those discussed in Remark 1, the claim on the Jacobian matrix $\nabla_{x} \psi_{z}(x, \xi)$ implies that all the components of the state of the original system (16) are represented in the chain of $n$ integrators.

Remark 4: By considering slight modifications to the construction in (19) and straightforward adaptation of the conditions $\left(C 1^{\prime}\right)$ and $\left(C 2^{\prime}\right)$, also nonlinear output functions $y=h(x)$ may be considered. As an explanatory example, consider the first two equations of system (7) and suppose that the output function is replaced by $y=h(x)=\sin \left(x_{1}\right)$, which is such that the relative degree is not well-defined at the origin. Then, in this context, $(C 1)$ holds since the matrix $\left[d h(0)^{\prime} d L_{f} h(0)^{\prime}\right]^{\prime}$, with $d h(x)=\left[\cos \left(x_{1}\right), 0\right]$ and

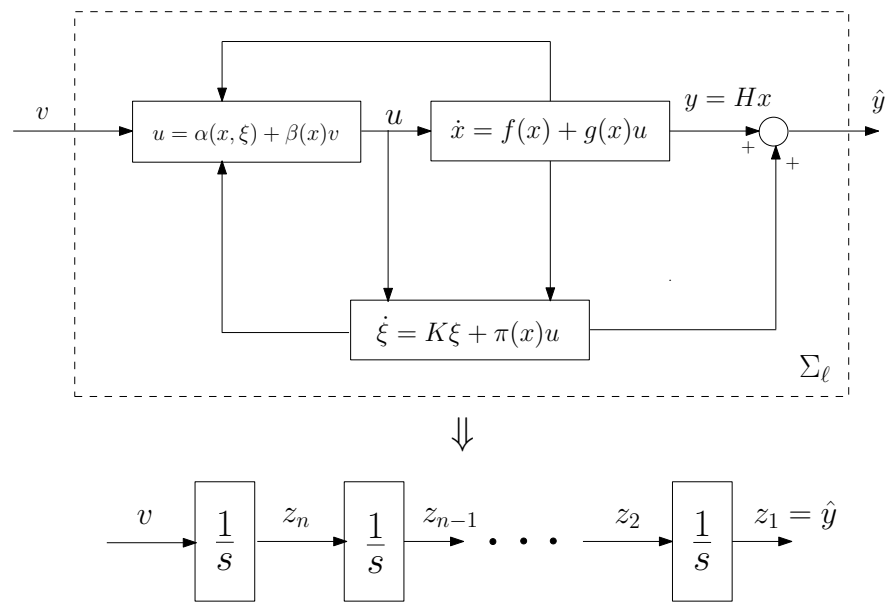

Fig. 1. Architecture of the closed-loop system (top graph) comprising the original nonlinear system and the dynamic extension, together with its linear input-output description (bottom graph).

$d L_{f} h(x)=\left[\sin \left(x_{1}\right)\left(x_{1}+x_{2}\right)+\cos \left(x_{1}\right), \cos \left(x_{1}\right)\right]$, has full rank, while $(C 2)$ is verified for any $\kappa \in \mathbb{R}$, since $L_{g} L_{f} h(x)=$ $\left(\sin \left(x_{1}\right)\left(x_{1}+x_{2}\right)+\cos \left(x_{1}\right)\right) x_{1}+\cos \left(x_{1}\right)$. Therefore, the construction discussed above can be carried out by defining the well-defined change of coordinates $z_{1}=\sin \left(x_{1}\right)+\xi_{1}$, $z_{2}=\cos \left(x_{1}\right)\left(x_{1}+x_{2}\right)+\xi_{2}$, together with the (local) inverse transformation $x_{1}=\arcsin \left(z_{1}-\xi_{1}\right)$ and $x_{2}=\left(z_{2}-\right.$ $\left.\xi_{2}\right) /\left(\cos \left(\arcsin \left(z_{1}-\xi_{1}\right)\right)\right)-\arcsin \left(z_{1}-\xi_{1}\right)$.

The following result, exploiting the construction in Proposition 2, employs Proposition 4 to provide a stabilizing feedback.

Proposition 5: Consider the nonlinear system (16). Suppose that there exist a matrix $H \in \mathbb{R}^{1 \times n}$ and constants $\kappa_{j} \in \mathbb{R}$, $j=1, \ldots, n-1$, such that $\left(C 1^{\prime}\right)$ and $\left(C 2^{\prime}\right)$ hold. Suppose in addition that $\left(C 3^{\prime}\right)$

1) the $(n-1) \times(n-1)$ matrix $\mathcal{A}_{\kappa}$ in (20) (top of the next page) is Hurwitz.

Consider system (16) in closed loop with (19), with

$$
\begin{aligned}
v= & -c_{1}\left(H x+\xi_{1}\right)-c_{2}\left(L_{f}(H x)+\xi_{2}\right)-\ldots \\
& \left.-c_{n}\left(L_{f}^{n-1}(H x)-\sum_{j=1}^{n-1} \kappa_{j} \xi_{j}\right)\right) .
\end{aligned}
$$

Then there exists a neighborhood $\mathcal{U}$ of $z_{o}=\psi_{z}(0,0)=0$ such that for any $\lambda \in \mathbb{R}_{+}$there exist $c_{i}, i=1, \ldots, n$, such that $\|z(t)\|<e^{-\lambda t}\|z(0)\|$ for all $z(0) \in \mathcal{U}$ and moreover the equilibrium point $(x, \xi)=(0,0)$ of (16)-(19)-(21) is locally asymptotically stable.

The proof of Proposition 5 employs arguments identical to those of the proof of Proposition 2 by requiring that the constants $c_{i}, i=1, \ldots, n$ be selected such that the polynomial $c_{1}+c_{2} s+c_{3} s^{2}+\ldots+c_{n} s^{n-1}+s^{n}$ is Hurwitz. Since the spectrum of $\mathcal{A}_{\kappa}$ consists of the union of $\Lambda\left(A_{u u}\right)$, namely the eigenvalues associated to the uncontrollable subsystem of the linearized system, and a subset that can be arbitrarily assigned via the choice of the coefficients $\kappa_{i}$, the sufficient condition $\left(C 3^{\prime}\right)$ cannot be verified if $\Lambda\left(A_{u u}\right) \subset \mathbb{C}^{+} \cup \mathbb{C}^{0}$. The scheme in Figure 1 allows to visualize the convergence properties that can be enforced on systems that are not feedback linearizable: the dynamic control law (19) guarantees stability and (local) exponential convergence of a desired output of the system 


$$
\begin{gathered}
\mathcal{A}_{\kappa}=\left[\begin{array}{ccccc}
0 & 1 & 0 & \ldots & 0 \\
0 & 0 & 1 & \ldots & 0 \\
\vdots & & & \ddots & \vdots \\
-\kappa_{1} & -\kappa_{2} & & \ldots & -\kappa_{n-1}
\end{array}\right]-\nabla_{\xi}\left[\begin{array}{c}
\left.L_{g}(H x) \alpha(x, \xi)\right|_{(x, \xi)=\psi^{-1}(z, \xi)} \\
\left.L_{g} L_{f}(H x) \alpha(x, \xi)\right|_{(x, \xi)=\psi^{-1}(z, \xi)} \\
\vdots \\
\left.L_{g} L_{f}^{n-3}(H x) \alpha(x, \xi)\right|_{(x, \xi)=\psi^{-1}(z, \xi)} \\
\left.L_{g} L_{f}^{n-2}(H x) \alpha(x, \xi)\right|_{(x, \xi)=\psi^{-1}(z, \xi)}
\end{array}\right]_{(z, \xi)=(0,0)} \\
\alpha(x, \xi)=\left(\begin{array}{c}
-L_{f}^{n}(H x)+\sum_{i=1}^{n-1} \kappa_{i} \kappa_{n-1} \xi_{i}-\sum_{j=1}^{n-2} \kappa_{j} \xi_{j+1} \\
\left.L_{g} L_{f}^{n-1}(H x)+\sum_{j=1}^{n-1} \kappa_{j} L_{g} L_{f}^{j-1}(H x)\right)^{-1}
\end{array}\right.
\end{gathered}
$$

towards a (locally) exponentially converging signal $\xi$. As in Section II-A, the control architecture can be exploited to enforce approximate tracking. To this end consider a reference signal $y_{d}$, together with its time derivatives $y_{d}^{(1)}, \ldots, y_{d}^{(n)}$, assumed well-defined. By introducing notation similar to that of Section II-A, let

$$
\begin{aligned}
v= & -c_{1}\left(H x+\xi_{1}-y_{d}\right)-c_{2}\left(L_{f}(H x)+\xi_{2}-y_{d}^{(1)}\right)-\ldots \\
& \left.-c_{n}\left(L_{f}^{n-1}(H x)-\sum_{j=1}^{n-1} \kappa_{j} \xi_{j}\right)-y_{d}^{(n-1)}\right) \\
& +y_{d}^{(n)} \triangleq \sigma(x, \xi, t)
\end{aligned}
$$

and let, $\bar{\eta}(z, \xi, t)$ denote the dynamics of the $\xi$-subsystem of (19) in the $(z, \xi)$-coordinates with $v=\sigma(x, \xi, t)$ and let $y_{R} \triangleq\left(y_{d}, \ldots, y_{d}^{(n-1)}\right)$ be a compact notation for $y_{d}$ and its time derivatives, which are continuous for all $t \geq 0$ and bounded, thus adapting Assumption 1 to the higher-dimensional case.

Proposition 6: Consider the nonlinear system (16) together with a given output $y=C x$. Suppose that there exist constants $\kappa_{j} \in \mathbb{R}, j=1, \ldots, n-1$, such that $C$ and $\kappa_{j}$ satisfy conditions $\left(C 1^{\prime}\right)$ and $\left(C 2^{\prime}\right)$, with $C$ in place of $H$. Consider system (16) in closed loop with (19), with $v$ selected as in (22) with $c_{i}$, $i=1, \ldots, n$ such that the polynomial $c_{1}+c_{2} s+c_{3} s^{2}+\ldots+$ $c_{n} s^{n-1}+s^{n}$ is Hurwitz. Suppose that the solution $\xi_{s s}$ of

$$
\dot{\xi}(t)=\bar{\eta}\left(y_{R}(t), \xi(t), t\right),
$$

with initial condition $\xi_{s s}(0)=0$, is defined for all $t \geq 0$, bounded and uniformly asymptotically stable. Then, for any $\varepsilon>0$ there exists $\delta>0$ such that $\left\|z_{i}(0)-y_{d}^{(i-1)}(0)\right\|<$ $\delta, i=1, \ldots, n,\left\|\xi_{j}(0)\right\|<\delta, j=1, \ldots, n-1$, imply that $\left\|z_{1}(t)-y_{d}(t)\right\|<\varepsilon$ and

$$
\left\|y(t)-y_{d}(t)\right\| \leq \varepsilon+\left\|\xi_{s s, 1}(t)\right\|,
$$

for all $t \geq 0$ and for any sufficiently small $y_{d}^{(i)}(t)$.

A comparison between the results of Proposition 6 and the approximate feedback linearization and tracking method based on the notion of robust relative degree of [11] is in order. Firstly, the latter concept and construction yield a perturbed chain of integrators, while the approach presented here provides an unperturbed chain of integrators with a perturbed output. The difference is visually summarized in Figure 2. This comparison is further expanded in the simulation section, in which we consider the model of the Ball and Beam system [11]. Moreover, as discussed in [5], the choice of the approximating functions of $x$ - which modify the canonical change of coordinates for feedback linearization - at each step in the construction of a robust relative degree (or the segregation of the functions into higher-order terms) are far from being obvious and may require complex algebraic manipulations. Such functions are, for instance, approximated using spline functions in [5], hence introducing an additional source of approximation. In the approach introduced here, this explicit choice of functions of the state can be completely circumvented, since it is replaced by functions of time, namely the states $\xi_{i}(t)$, the time evolutions of which are not imposed a priori, but actually determined in closed loop by the current value of the state (via the choice of $\dot{\xi}_{i}$ ): the resulting evolution of the state $\xi(t)$, driven by $x(t)$, behaves (in terms of input/output properties of the closed-loop system) as the functions required in [11], which however must not be known a priori here.
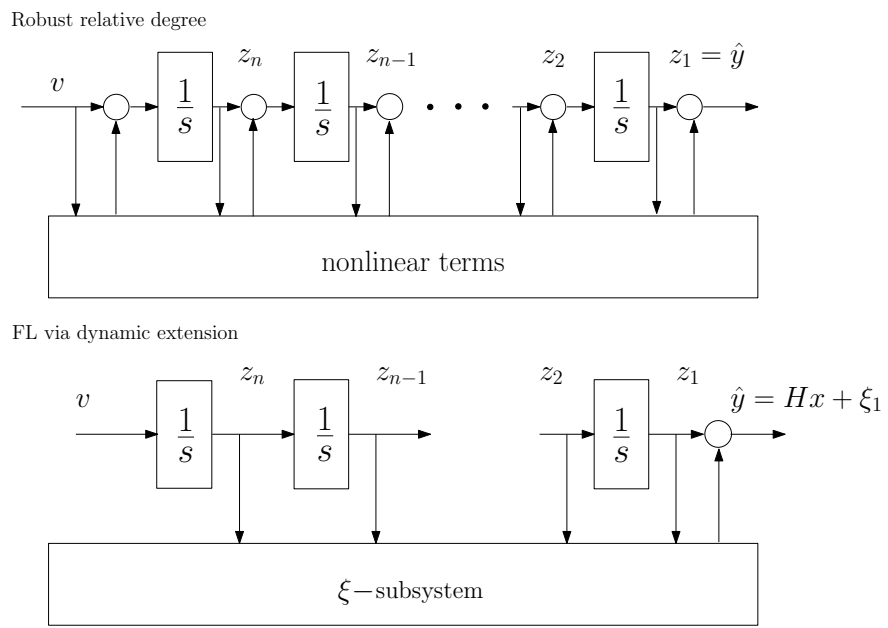

Fig. 2. Comparison between the method based on the notion of robust relative degree and the proposed approximate feedback linearization via dynamic extension.

\section{Design of ObSERVERS With Approximate LINEAR ERROR DYNAMICS}

The second tool required for output feedback design consists in an observer, which allows the reconstruction of the components of the state $x$ that are not directly measured. Such an observer design problem is the topic of this section. 
In particular, the construction proposed herein is carried out by combining the approximation ideas discussed above in the case of feedback design together with the theory concerning the so-called Observer Linearization Problem [13], recalled below. To this end, consider autonomous nonlinear systems with output described by equations of the form

$$
\begin{aligned}
& \dot{x}=f(x), \\
& y=h(x),
\end{aligned}
$$

with $x(t) \in \mathbb{R}^{n}$ and $y(t) \in \mathbb{R}$. Assume, without loss of generality, that the system (25) - with sufficiently smooth vector field $f: \mathbb{R}^{n} \rightarrow \mathbb{R}^{n}$ and output function $h: \mathbb{R}^{n} \rightarrow \mathbb{R}$ - possesses an equilibrium at the origin, namely $f(0)=0$, and $h(0)=0$. Moreover, we suppose that the trajectories of the system (25) are uniquely defined for all $t \geq 0$. The derivations are initially limited to autonomous systems and then extended to the case of systems with input in Section IIIB. The construction detailed in the following results provides a local observer according to the definition below.

Definition 1: A dynamical system

$$
\begin{aligned}
& \dot{\chi}=\sigma(\chi, y), \\
& \hat{x}=\vartheta(\chi, y),
\end{aligned}
$$

with $\chi(t) \in \mathbb{R}^{n_{\chi}}$ and $\hat{x}(t) \in \mathbb{R}^{n}$, is said to be a local observer for system (25) if system (25)-(26) satisfies the two following requirements. (i)

1) If $x(0)=\hat{x}(0)$, then $x(t)=\hat{x}(t)$ for all $t \geq 0$.

2) There exists a neighborhood $\mathcal{U}$ of the origin of $\mathbb{R}^{n} \times \mathbb{R}^{n_{\chi}}$ such that for all $(x(0), \chi(0)) \in \mathcal{U}$, the estimation error $\hat{x}(t)-x(t)$ decays asymptotically to zero.

Moreover, the local observer is said to be spectrally assignable if for any $\alpha>0$, there exist $\sigma, \vartheta$ and $c>0$ such that $\|x(t)-\hat{x}(t)\| \leq c e^{-\alpha t}\|x(0)-\hat{x}(0)\|$ for all $t \geq 0$ and all $(x(0), \chi(0)) \in \mathcal{U}$.

\section{A. Planar Systems}

Mimicking the architecture of the previous sections, the observer design strategy is initially discussed in the case of planar systems, in order to highlight the rationale behind its construction. To this end consider the following assumption.

Assumption 2: The nonlinear system (25), with $n=2$, satisfies the observability rank condition at the origin, i.e.

$$
\operatorname{rank}\left(\mathrm{d} \mathcal{O}(x)_{x=0}\right) \triangleq \operatorname{rank}\left(\left[\begin{array}{c}
d h(x) \\
d L_{f} h(x)
\end{array}\right]_{x=0}\right)=2 .
$$

To put the following derivations into perspective it is worth briefly recalling the task that has been referred to as the Observer Linearization Problem in [13], which can be considered as a natural counterpart to the Exact Linearization via Feedback Problem dealt with in Section II. The challenge consists in designing observers that yield a linear and spectrally assignable error dynamics, possibly modulo a change of coordinates. In [13, Lemma 4.9.2] it is shown that the Observer Linearization Problem is solvable if and only if the nonlinear system satisfies the observability rank condition and a certain partial differential equation admits a solution, see [13, eq.
(4.74)]. The result is then constructively achieved by defining a change of coordinates $z=\Phi(x)$ that transforms system (25) into $\dot{z}=A z+k(y), y=C z$, with the pair $(C, A)$ observable. Such coordinates transformation is essentially related to a modified version of the classical change of coordinates derived from the output function $h$ and its repeated Lie derivatives along the vector field $f$, which however involve the solution of the partial differential equation mentioned above. As in the previous sections the main objective here is to approximate such construction circumventing the explicit computation of the transformation - which may not even exist - by introducing a dynamic extension driven by a copy of the state of the system that replicates the effect of the desired change of coordinates in the transformed state-space. To provide a concise statement consider the change of coordinates defined by

$$
\left[\begin{array}{l}
z \\
\xi
\end{array}\right]=\left[\begin{array}{c}
\phi_{z}(x, \xi) \\
\xi
\end{array}\right]=\left[\begin{array}{c}
h(x)+\xi_{1} \\
L_{f} h(x)+\xi_{2} \\
\xi
\end{array}\right] \triangleq \phi(x, \xi),
$$

which is a well-defined local diffeomorphism by Assumption 2, with the dynamics of $\xi(t)=\left[\xi_{1}(t), \xi_{2}(t)\right]^{\top} \in \mathbb{R}^{2}$ to be assigned. Let the function $L_{f}^{2} h(x)$ be partitioned $a^{2}$ $L_{f}^{2} h(x)=\varphi_{2,1}(y)+\varphi_{2,2}(x)$. Finally, let $P=\left[\begin{array}{ll}0 & 1\end{array}\right]^{\top}$, $M \in \mathbb{R}^{1 \times 2}$ and $N \in \mathbb{R}^{1 \times 2}$ denote the linear components of the function $\varphi_{2,2}\left(\phi^{-1}(z, \xi)\right)$ with respect to $z$ and $\xi$, respectively, i.e. $M=\nabla_{z}\left(\varphi_{2,2}\left(\phi^{-1}(z, \xi)\right)\right)_{(z, \xi)=(0,0)}$ and $N=\nabla_{\xi}\left(\varphi_{2,2}\left(\phi^{-1}(z, \xi)\right)\right)_{(z, \xi)=(0,0)}$, while $\bar{\varphi}_{2,2}(z, \xi)=$ $\varphi_{2,2}\left(\phi^{-1}(z, \xi)\right)-N \xi$ and $\tilde{\varphi}_{2,2}(z, \xi)=\varphi_{2,2}\left(\phi^{-1}(z, \xi)\right)-$ $N \xi-M z$. The following assumption characterizes the set of admissible trajectories of system (25).

Assumption 3: There exists a compact set $\mathcal{X} \subset \mathbb{R}^{2}$ such that $x(0) \in \mathcal{X}$ implies $x(t) \in \mathcal{X}$ for all $t \geq 0$, namely $\mathcal{X}$ is a forward invariant set for system (25).

Proposition 7: Consider the nonlinear system (25) and suppose that Assumptions 2 and 3 hold. Consider the dynamical system with input $y$ and output $\hat{x}$ defined as

$$
\begin{aligned}
& \dot{\xi}=F \xi-P \bar{\varphi}_{2,2}(\hat{z}, \xi), \\
& \dot{\hat{z}}=A_{o} \hat{z}+P \varphi_{2,1}(y)+\Gamma \xi+G\left(y+\xi_{1}-C_{o} \hat{z}\right), \\
& \hat{x}=\phi^{-1}(\hat{z}, \xi)
\end{aligned}
$$

with $(\xi(t), \hat{z}(t)) \in \mathbb{R}^{2} \times \mathbb{R}^{2}$,

$$
F=\left[\begin{array}{cc}
0 & 1 \\
-\gamma_{1} & -\gamma_{2}
\end{array}\right], \quad A_{o}=\left[\begin{array}{ll}
0 & 1 \\
0 & 0
\end{array}\right], \quad C_{o}=\left[\begin{array}{ll}
1 & 0
\end{array}\right]
$$

and

$$
\Gamma=P N+\left[\begin{array}{cc}
0 & 0 \\
-\gamma_{1} & -\gamma_{2}
\end{array}\right]
$$

Then there exist constants $\gamma_{1}>0, \gamma_{2}>0$ and a matrix $G$ such that

i) system (29) is a spectrally assignable local observer for system (25);

\footnotetext{
${ }^{2}$ This partition is not essential to the following derivations, however it is interestingly inspired by the spirit of the observer linearization problem. Provided the output function $h$ and the mapping $f$ are polynomial, or analytic, functions, it can be achieved by performing an iterated sequence of polynomial divisions of the function $L_{f}^{2} h(x)$ by the polynomial $h(x)$.
} 
ii) the transfer functions from the input $v=-\bar{\varphi}_{2,2}(\hat{z}, \xi)$ to the outputs $\xi_{i}, i=1,2$, of system (29a) do not possess finite zeros for all $s \in \mathbb{C} \backslash\{0\}$.

Proof: The claim is proved in two steps: first, the description of the system in the transformed $(z, \xi)$-coordinates is derived by considering the dynamics in (29a), then it is shown that (29b) provides a linear and spectrally assignable observer for system (25), while item $i i$ ) of the statement follows directly from the structure of the dynamics (29a). Thus, consider the first component $z_{1}=h(x)+\xi_{1}$, which yields

$$
\dot{z}_{1}=L_{f} h(x)+\dot{\xi}_{1}=L_{f} h(x)+\xi_{2}=z_{2},
$$

while $z_{2}=L_{f} h(x)+\xi_{2}$ yields

$$
\begin{aligned}
\dot{z}_{2} & =L_{f}^{2} h(x)+\dot{\xi}_{2} \\
& =\varphi_{1}(y)+\bar{\varphi}_{2,2}(z, \xi)+N \xi-\gamma_{1} \xi_{1}-\gamma_{2} \xi_{2}-\bar{\varphi}_{2,2}(\hat{z}, \xi) .
\end{aligned}
$$

Combining the two previous equations yields

$$
\dot{z}=A_{o} z+P \varphi_{1}(y)+\Gamma \xi+P\left(\bar{\varphi}_{2,2}(z, \xi)-\bar{\varphi}_{2,2}(\hat{z}, \xi)\right),
$$

$$
y=C_{o} z-\xi_{1}
$$

where the output expression is derived directly from the definition of $z_{1}$ and of the matrix $C_{o}$. Note that the pair $\left(C_{o}, A_{o}\right)$ is observable. Defining the error as $e=z-\hat{z}$, the corresponding dynamics are given by

$$
\begin{aligned}
\dot{e} & =A_{o} e-G C_{o} e+P\left(\bar{\varphi}_{2,2}(z, \xi)-\bar{\varphi}_{2,2}(\hat{z}, \xi)\right) \\
& =\left(A_{o}-G C_{o}\right) e+P\left(\tilde{\varphi}_{2,2}(z, \xi)+M z-\tilde{\varphi}_{2,2}(\hat{z}, \xi)-M \hat{z}\right) \\
& =\left(A_{o}+P M-G C_{o}\right) e+P\left(\tilde{\varphi}_{2,2}(z, \xi)-\tilde{\varphi}_{2,2}(\hat{z}, \xi)\right) .
\end{aligned}
$$

The first claim is proved by noting that observability of the pair $\left(C_{o}, A_{o}\right)$ implies observability of the pair $\left(C_{o}, A_{o}+P M\right)$, by the structure of $P$, hence there exist sufficiently large positive constants $\gamma_{1}$ and $\gamma_{2}$ in $F$ and matrix gain $G$ such that the zero equilibrium of the unforced, i.e. with $y=0,(\xi, \hat{z})$-dynamics is locally asymptotically stable. Together with Assumption 3 , the latter property implies that $\xi$ and $\hat{z}$ are bounded for all time along the trajectories of the closed-loop system and the conclusions follow by smoothness of the function $\tilde{\varphi}_{2,2}$.

Remark 5: By inspecting the proof of Proposition 7, it is evident that the gains $\gamma_{i}, i=1,2$, and the matrix $G$ should be constructively selected such that $\Gamma$ and $\left(A_{o}-P M-G C_{o}\right)$, respectively, are Hurwitz.

Remark 6: The structure of the transformed dynamics (30) is reminiscent of that achieved by the exact solution to the observer linearization problem, however with the presence of terms containing the dynamic extension $\xi$ that compensates for the non-exact knowledge (or the non-existence) of the appropriate change of coordinates.

Remark 7: By inspecting the structure of the observer (29), it is interesting to point out that the change of coordinates $z_{1}=h(x)+\xi_{1}, z_{2}=L_{f} h(x)+\xi_{2}$ essentially consists of a combination of the output and its derivative namely $h(x)$, $L_{f} h(x)$, and of its repeated integrals, implicitly performed by the choice of the dynamics of $\xi$. Such dynamics are in fact provided by a chain of integrators driven by the state $\hat{z}$, which is in turn driven by the output $y$.

The importance of item $i$ ) of the claims of Proposition 7 is the topic of the following remark, in which the difference with respect to classical high-gain observers is highlighted: it is essentially related to the fact that the poles of the transfer function excited by the nonlinear components of the observer (29a)-(29b) can be arbitrarily tuned without affecting its zeros, while the zeros of standard high-gain observers are placed by the same parameters that assign the poles.

Remark 8: Consider a planar nonlinear system described by equations of the form (25). Then, the high-gain observer design consists in defining the change of coordinates $z_{1}^{h}=$ $h(x), z_{2}^{h}=L_{f} h(x)$, which is well-defined by Assumption 2, yielding the transformed dynamics

$$
\begin{aligned}
\dot{z}_{1}^{h} & =z_{2}^{h}, \\
\dot{z}_{2}^{h} & =L_{f}^{2} h\left(\phi_{h}^{-1}\left(z^{h}\right)\right) \triangleq \varrho\left(z^{h}\right), \\
y & =z_{1}^{h} .
\end{aligned}
$$

The observer dynamics are then defined as

$$
\begin{aligned}
\dot{z}_{1}^{h} & =\hat{z}_{2}^{h}+\frac{k_{1}^{h}}{\varepsilon}(y-\hat{y}), \\
\dot{z}_{2}^{h} & =\varrho\left(\hat{z}^{h}\right)+\frac{k_{2}^{h}}{\varepsilon^{2}}(y-\hat{y}), \\
\hat{y} & =\hat{z}_{1}^{h},
\end{aligned}
$$

while the estimate is provided by $\hat{x}=\phi_{h}^{-1}(\hat{z})$. The equations (33) can be written in compact form as

$$
\dot{\hat{z}}^{h}=A_{h} \hat{z}^{h}+P \varrho\left(\hat{z}^{h}\right)+B_{h} y,
$$

with

$$
A_{h}=\left[\begin{array}{cc}
-\frac{k_{1}^{h}}{\varepsilon} & 1 \\
-\frac{k_{2}^{h}}{\varepsilon^{2}} & 0
\end{array}\right], \quad B_{h}=\left[\begin{array}{c}
\frac{k_{1}^{h}}{\varepsilon} \\
\frac{k_{2}^{h}}{\varepsilon^{2}}
\end{array}\right] .
$$

A significant difference between (29) and (34) can be now identified. Towards this end, by inspecting (34) and the structure of $A_{h}$ and $B_{h}$ it is evident that the degree of freedom available to compensate for the presence of the nonlinear term $\varrho$ - decreasing the value of $\varepsilon$ - has the simultaneous effect of increasing the reliability on the output $y$ : this feature may have critically detrimental consequences in the presence of noisy measurements. This is not the case, instead, in (29), in which the choice of the matrix $F$ that dominates the nonlinear terms is not necessarily a priori related to the matrix $G$ that multiplies the output signal $y$. This is achieved by introducing the copy of the nonlinear terms, to be compensated in the error dynamics, directly at the stage of the change of coordinates, by means of the dynamic extension $\xi$, rather than in the dynamics of the observer, as in (34).

\section{B. General Nonlinear systems}

The extension of the observer design method above to higher-dimensional systems is the objective of this section. Let $L_{f}^{n} h(x)=\varphi_{n, 1}(y)+\varphi_{n, 2}(x)$ and consider for $\varphi_{n, 2}(x)$ notation similar to the one introduced around $\varphi_{2,2}(x)$ for the matrices $M \in \mathbb{R}^{1 \times n}$ and $N \in \mathbb{R}^{1 \times n}$ and the functions 
$\bar{\varphi}_{n, 2}$ and $\tilde{\varphi}_{n, 2}$, i.e. $M=\nabla_{z}\left(\varphi_{n, 2}\left(\phi^{-1}(z, \xi)\right)\right)_{(z, \xi)=(0,0)}$, $N=\nabla_{\xi}\left(\varphi_{n, 2}\left(\phi^{-1}(z, \xi)\right)\right)_{(z, \xi)=(0,0)}, \quad \bar{\varphi}_{n, 2}(z, \xi)=$ $\varphi_{n, 2}\left(\phi^{-1}(z, \xi)\right)-N \xi$ and $\tilde{\varphi}_{n, 2}(z, \xi)=\varphi_{n, 2}\left(\phi^{-1}(z, \xi)\right)-$ $N \xi-M z$.

Assumption 4: There exists a compact set $\mathcal{X} \subset \mathbb{R}^{n}$ such that $x(0) \in \mathcal{X}$ implies $x(t) \in \mathcal{X}$ for all $t \geq 0$, namely $\mathcal{X}$ is a forward invariant set for system (25).

Suppose, in addition, that the following assumption, which generalizes Assumption 2, holds.

Assumption 5: The nonlinear system (25) satisfies the $o b$ servability rank condition at the origin, namely

$$
\operatorname{rank}\left(\mathrm{d} \mathcal{O}(x)_{x=0}\right) \triangleq \operatorname{rank}\left(\left[\begin{array}{c}
d h(x) \\
d L_{f} h(x) \\
\vdots \\
d L_{f}^{n-1} h(x)
\end{array}\right]_{x=0}\right)=n .
$$

Finally, consider the change of coordinates defined by

$$
\left[\begin{array}{c}
z \\
\xi
\end{array}\right]=\left[\begin{array}{c}
\psi_{z}(x, \xi) \\
\xi
\end{array}\right]=\left[\begin{array}{c}
h(x)+\xi_{1} \\
L_{f} h(x)+\xi_{2} \\
\vdots \\
L_{f}^{n-1} h(x)+\xi_{n} \\
\xi
\end{array}\right] \triangleq \psi(x, \xi)
$$

with $\xi \in \mathbb{R}^{n}$.

Proposition 8: Consider the nonlinear system (25) and suppose that Assumptions 4 and 5 hold. Consider the dynamical system with output defined as

$$
\begin{aligned}
& \dot{\xi}=F \xi-P \bar{\varphi}_{n, 2}(\hat{z}, \xi), \\
& \dot{\hat{z}}=A_{o} \hat{z}+P \varphi_{n, 1}(y)+\Gamma \xi+G\left(y+\xi_{1}-C_{o} \hat{z}\right), \\
& \hat{x}=\psi^{-1}(\hat{z}, \xi)
\end{aligned}
$$

with $(\xi(t), \hat{z}(t)) \in \mathbb{R}^{n} \times \mathbb{R}^{n}$,

$$
\begin{aligned}
& F=\left[\begin{array}{ccccc}
0 & 1 & \ldots & 0 & 0 \\
0 & 0 & 1 & \ldots & 0 \\
\vdots & \vdots & \vdots & \ddots & \vdots \\
0 & 0 & 0 & \ldots & 1 \\
-\gamma_{1} & -\gamma_{2} & \ldots & -\gamma_{n-1} & -\gamma_{n}
\end{array}\right] \\
& P=\left[\begin{array}{llll}
0 & 0 & \ldots & 1
\end{array}\right]^{\top} \text {, } \\
& A_{o}=\left[\begin{array}{ccccc}
0 & 1 & \ldots & 0 & 0 \\
0 & 0 & 1 & \ldots & 0 \\
\vdots & \vdots & \vdots & \ddots & \vdots \\
0 & 0 & 0 & \ldots & 1 \\
0 & 0 & \ldots & 0 & 0
\end{array}\right] \\
& C_{o}=\left[\begin{array}{llll}
1 & 0 & \ldots & 0
\end{array}\right] \text {, and } \\
& \Gamma=P N+\left[\begin{array}{ccc}
0 & \cdots & 0 \\
\vdots & \ddots & \vdots \\
-\gamma_{1} & \cdots & -\gamma_{n}
\end{array}\right] \text {. }
\end{aligned}
$$

Then, there exist constants $\gamma_{i}>0, i=1, \ldots, n$, and a matrix $G$ such that i) system (37) is a spectrally assignable local observer for system (25);

ii) the transfer functions from the input $v=-\bar{\varphi}_{n, 2}(\hat{z}, \xi)$ to the outputs $\xi_{i}, i=1, \ldots, n$, of system (37a) do not possess finite zeros for all $s \in \mathbb{C} \backslash\{0\}$.

\section{Observer design for the Van der Pol's oscillator}

To further substantiate the discussion in Remark 8, the observer design problem for the Van der Pol's oscillator is solved in this section. Consider the Van der Pol's oscillator described by the equations

$$
\begin{aligned}
\dot{x}_{1} & =x_{2}, \quad \dot{x}_{2}=-x_{1}+\left(1-x_{1}^{2}\right) x_{2}, \\
y & =x_{1} .
\end{aligned}
$$

In the following simulations we suppose, similarly to [2], that the output is corrupted by high-frequency additive noise, modeled by $\nu(t)=\sin (\omega t)$, namely $\tilde{y}(t)=y(t)+\nu(t)$. Considering system (38), the change of coordinates (28) becomes $z_{1}=x_{1}+\xi_{1}, z_{2}=x_{2}+\xi_{2}$, and the dynamics of (29) reduces to

$$
\begin{aligned}
& \dot{\xi}_{1}=\xi_{2}, \\
& \dot{\xi}_{2}=-\gamma_{1} \xi_{1}-\gamma_{2} \xi_{2}-M \hat{z}+\left(\hat{z}_{1}-\xi_{1}\right)^{2}\left(\hat{z}_{2}-\xi_{2}\right), \\
& \dot{\hat{z}}_{1}=\hat{z}_{2}+g_{1}\left(\tilde{y}+\xi_{1}-\hat{z}_{1}\right), \\
& \dot{\hat{z}}_{2}=\left(1-\gamma_{1}\right) \xi_{1}-\left(1+\gamma_{2}\right) \xi_{2}+g_{2}\left(\tilde{y}+\xi_{1}-\hat{z}_{1}\right),
\end{aligned}
$$

with $M=\left[\begin{array}{ll}-1 & 1\end{array}\right]$, and $\hat{x}=\hat{z}-\xi$. The above observer is then compared with the standard high-gain observer introduced in Remark 8, which are sensitive to measurement noise but, on the other hand, robust with respect to model uncertainties. In particular, the comparison is initially performed by considering $\varepsilon=0.5$ in the high-gain observer and the constants $\gamma_{1}$ and $\gamma_{2}$ assigning the eigenvalues of the matrices $F$ and $A_{o}-G C_{o}$ at $\{-1,-2\}$. The time histories of the state of the corresponding error dynamics are depicted in Figure 3, showing similar attenuation properties for the two observers. Suppose now that one is interested in increasing the attenuation properties of the observer on the error dynamics. This is achieved, as illustrated in Figure 4, for the observer (39) by moving the eigenvalues of the matrix $F$ to $\{-40,-41\}$, while leaving those of the matrix $A_{o}-G C_{o}$ at $\{-1,-2\}$. A similar attempt is carried out also for the high-gain observer, by varying the gain $\varepsilon$, as depicted in Figures 5 and 6 with $\varepsilon=0.1$ and $\varepsilon=1$, respectively, showing that the high-gain observer is not capable of achieving a similar attenuation performance, at least in its basic implementation. Moreover, it is interesting to point out that the two-degree-of-freedom nature of the observer in (37), i.e. the independent choice of the gain matrices $F$ and $G$, renders the observer robust to model uncertainties, without compromising the ability of rejecting measurement noise. This feature is highlighted by the numerical simulation in Figure 7 , in which an additional term of the form $x_{1} x_{2}$ is introduced into $\dot{x}_{2}$ as unmodeled dynamics. Finally, it can be shown that the van der Pol's oscillator (38) satisfies the assumptions of [13, Lemma 4.9.2], hence an observer with linear error dynamics 


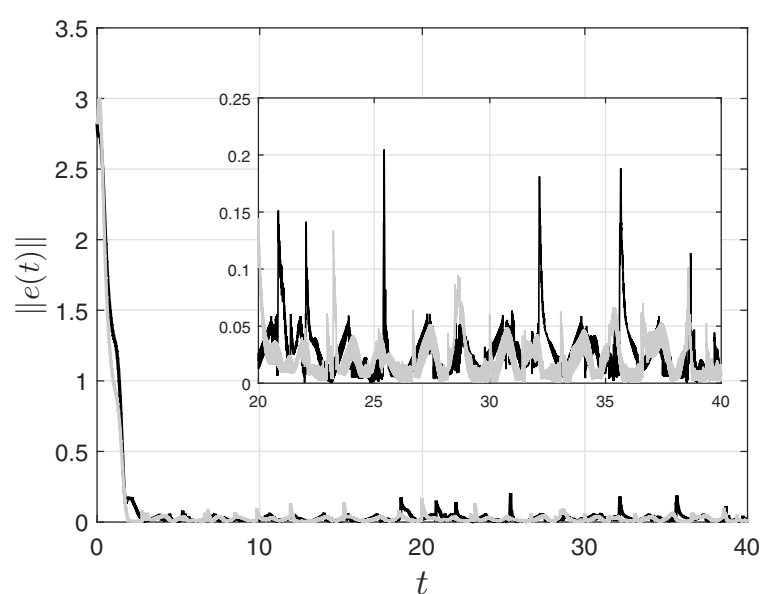

Fig. 3. Time histories of the state of the error dynamics provided by the observer (39), with the eigenvalues of the matrices $\boldsymbol{F}$ and $\boldsymbol{A}_{o}-\boldsymbol{G} \boldsymbol{C}_{o}$ at $\{-1,-2\}$ (black line) and of the high-gain observer (33) with $\varepsilon=0.5$ (gray line).

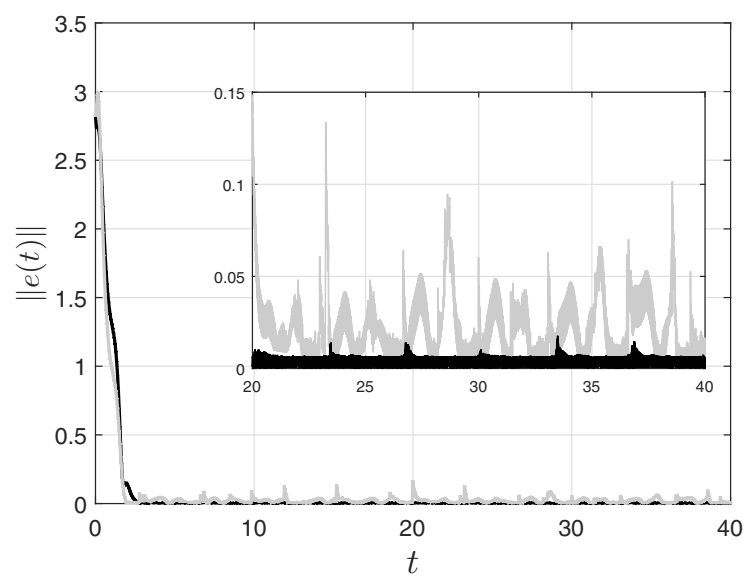

Fig. 4. Time histories of the error provided by the observer (39), with the eigenvalues of $F$ and $A_{o}-G C_{o}$ at $\{-40,-41\}$ and $\{-1,-2\}$, respectively, (black line) and of the high-gain observer (33) with $\varepsilon=\mathbf{0 . 5}$ (gray line).

can be constructed. This is achieved by means of the change of coordinates $z_{1}^{\ell}=x_{1}, z_{2}^{\ell}=x_{2}+x_{1}^{3} / 3$, yielding

$$
\dot{z}^{\ell}=\left[\begin{array}{ll}
0 & 1 \\
0 & 0
\end{array}\right] z^{\ell}-\frac{1}{3}\left[\begin{array}{l}
y^{3} \\
y^{3}
\end{array}\right] \triangleq A_{o} z^{\ell}-\beta(y) .
$$

An observer for system (40) can be then designed as $\dot{\hat{z}}^{\ell}=$ $A_{o} \hat{z}^{\ell}-\beta(y)-G\left(y-C_{o} \hat{z}^{\ell}\right)$, with the matrix $G$ such that $A_{o}-$ $G C_{o}$ is Hurwitz. Note, however, that such an observer suffers the same drawback of the high-gain design in the presence of measurement noise, i.e. whenever $\tilde{y}(t)=y(t)+\nu(t)$. In fact, letting $e^{\ell}=z^{\ell}-\hat{z}^{\ell}$, the error dynamics becomes $\dot{e}^{\ell}=\left(A_{o}-G C_{o}\right)+G \nu(t)$, where the matrix $G$ - which should be selected to dominate the noise assigning the eigenvalues of $\left(A_{o}-G C_{o}\right)$ - multiplies the noise itself. This issue is completely circumvented by the systematic design of the twodegree-of-freedom structure proposed in (37), at the price of an augmented dimension of the observer.

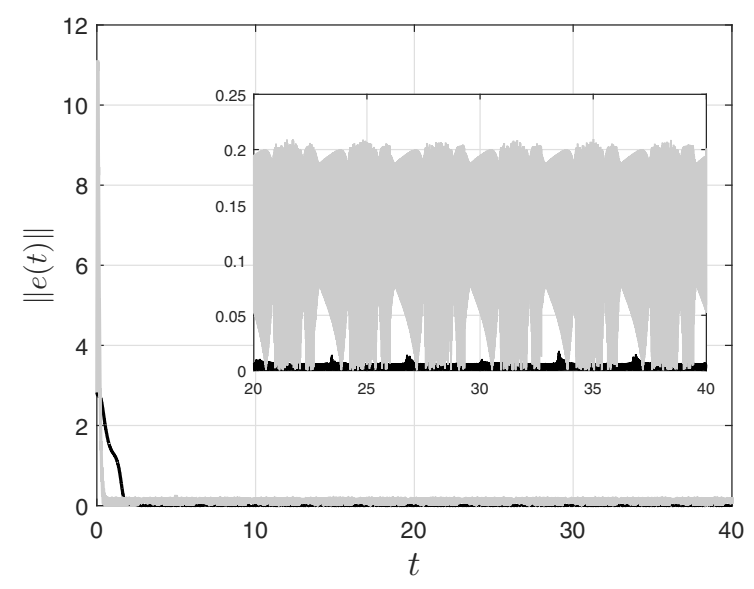

Fig. 5. Time histories of the error provided by the observer (39), with the eigenvalues of $F$ and $A_{o}-G C_{o}$ at $\{-40,-41\}$ and $\{-1,-2\}$, respectively, (black line) and of the high-gain observer (33) with $\varepsilon=\mathbf{0 . 1}$ (gray line).

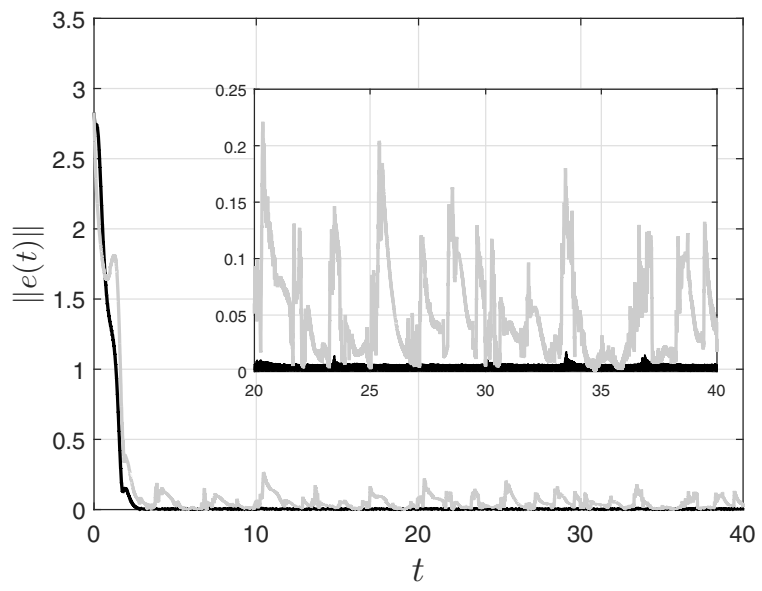

Fig. 6. Time histories of the error provided by the observer (39), with the eigenvalues of $\boldsymbol{F}$ and $\boldsymbol{A}_{o}-G C_{o}$ at $\{-40,-41\}$ and $\{-1,-2\}$, respectively, (black line) and of the high-gain observer (33) with $\varepsilon=1$ (gray line).

\section{A NONLINEAR SEPARATION PRINCIPLE}

In this section the two previously developed techniques inspired by feedback linearization and observers with linear error dynamics, respectively, are combined to design an output feedback stabilizing control law for a class of nonlinear systems. Consider controlled nonlinear systems with output described by equations of the form

$$
\begin{aligned}
\dot{x} & =f(x)+g(C x) u, \\
y & =C x,
\end{aligned}
$$

with $f: \mathbb{R}^{n} \rightarrow \mathbb{R}^{n}, g: \mathbb{R} \rightarrow \mathbb{R}^{n}$ and $C \in \mathbb{R}^{1 \times n}$.

\section{A. Planar Systems}

Mimicking the structure of the previous sections the discussion is initially carried out in the case of planar nonlinear systems and then extended to higher-order systems. To begin 


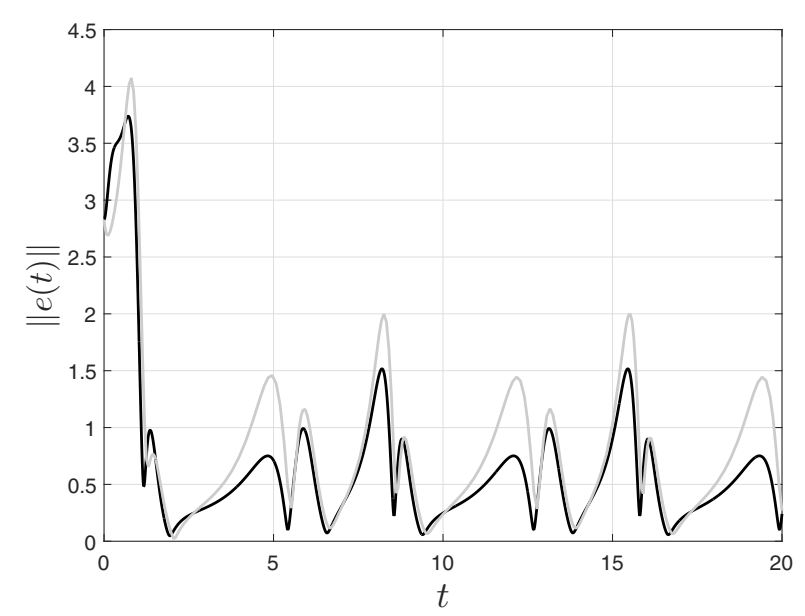

Fig. 7. Time histories of the error provided by the observer (39), with the eigenvalues of $F$ and $A_{o}-G C_{o}$ at $\{-40,-41\}$ and $\{-1,-2\}$, respectively, (black line) and of the high-gain observer (33) with $\varepsilon=1$ (gray line), in the presence of unmodeled dynamics in $\dot{x}_{2}$.

with, note that, by linearity of the output function, Assumption 5 implies that the matrix $C$ satisfies the condition $(C 1)$ of Proposition 4, with $C$ in place of $H$. Therefore, assuming that condition $(C 2)$ holds for $C$, the change of coordinates

$$
\left[\begin{array}{c}
z \\
\xi_{c}
\end{array}\right]=\left[\begin{array}{c}
\psi_{z}\left(x, \xi_{c}\right) \\
\xi_{c}
\end{array}\right]=\left[\begin{array}{c}
C x+\xi_{c} \\
L_{f}(C x)-\kappa \xi_{c} \\
\xi_{c}
\end{array}\right] \triangleq \psi\left(x, \xi_{c}\right),
$$

is a well-defined local diffeomorphism. In the transformed coordinates, the system (41a) becomes

$$
\begin{aligned}
& \dot{z}_{1}=z_{2}, \\
& \dot{z}_{2}=L_{f}^{2}(C x)+\kappa^{2} \xi_{c}+\left(L_{g} L_{f}(C x)+\kappa L_{g}(C x)\right) u,
\end{aligned}
$$

together with the output $y=C_{o} z-\xi_{c}$ and the dynamics of $\xi_{c}$ defined as $\dot{\xi}_{c}=-\kappa \xi_{c}-C g(y) u$. Let $\alpha\left(z, \xi_{c}\right)=$ $\left(L_{f}^{2}(C x)+\kappa^{2} \xi_{c}\right)_{\left(x, \xi_{c}\right)=\psi^{-1}\left(z, \xi_{c}\right)}$ and $\beta\left(z, \xi_{c}\right)=\left(L_{g} L_{f}(C x)+\right.$ $\left.\kappa L_{g}(C x)\right)_{\left(x, \xi_{c}\right)=\psi^{-1}\left(z, \xi_{c}\right)}$. It is evident that, defining the function $\gamma: \mathbb{R}^{2} \times \mathbb{R} \times \mathbb{R} \rightarrow \mathbb{R}$ as

$$
\gamma\left(z, \xi_{c}, \bar{u}\right)=-\left(\alpha\left(z, \xi_{c}\right)-\bar{u}\right) \beta\left(z, \xi_{c}\right)^{-1},
$$

then, by applying the control law $u^{\star}=\gamma\left(z, \xi_{c}, K z\right)$, with $K=\left[k_{1}, k_{2}\right]$ such that $\Lambda\left(A_{o}+B_{o} K\right) \subset \mathbb{C}^{-}$, with $A_{o}$ defined above and $B_{o}=\left[\begin{array}{ll}0,1\end{array}\right]^{\top}$, the origin of the system (43) becomes an exponentially stable equilibrium point. On the other hand, the implementation of the control law $u=$ $\gamma\left(\hat{z}, \xi_{c}, \bar{u}\right)$, with $\hat{z}$ to be defined, yields

$$
\dot{z}=A_{o} z+B_{o} \bar{u}+P\left(\delta\left(z, \xi_{c}, \hat{z}, \bar{u}\right)-\delta\left(z, \xi_{c}, z, \bar{u}\right)\right),
$$

with $P=\left[\begin{array}{ll}0,1\end{array}\right]^{\top}$ and $\delta\left(z, \xi_{c}, \hat{z}, \bar{u}\right)=\beta\left(z, \xi_{c}\right) \gamma\left(\hat{z}, \xi_{c}, \bar{u}\right)$. To streamline the statement of the following result, define the estimation error $e=z-\hat{z}$ and let the matrix $M$ and the function $\varphi$ be such that $\delta(\cdot, \cdot, z, \cdot)=M z+\tilde{\delta}(\cdot, \cdot, z, \cdot)$, with $\left.\nabla_{z} \tilde{\delta}\left(\lambda_{1}, \lambda_{2}, z, \lambda_{3}\right)\right|_{\left(\lambda_{1}, \lambda_{2}, z, \lambda_{3}\right)=0}=0$, hence separating the linear terms in $z$ of $\delta$. Finally, let the variable $v$ denote the nonlinear input mismatch, namely

$$
v=\tilde{\delta}\left(z, \xi_{c}, \hat{z}, \bar{u}\right)-\tilde{\delta}\left(z, \xi_{c}, z, \bar{u}\right),
$$

i.e. the difference between the state feedback stabilizing control law and the certainty equivalence implementation.

Proposition 9: Consider the system (41) and suppose that there exists $\kappa$ such that condition $(C 2)$ of Proposition 1 holds, with $C$ in place of $H$. Let $\bar{u}=K \hat{z}$, with $K$ such that $\Lambda\left(A_{o}+\right.$ $\left.B_{o} K\right) \subset \mathbb{C}^{-}$and $G$ such that $\Lambda\left(A_{o}-P M-G C_{o}\right) \subset \mathbb{C}^{-}$. Then, in the $\left(\xi_{c}, z, e\right)$ coordinates, the system (41) in closed loop with

$$
\begin{aligned}
u & =-\left(\alpha\left(\hat{z}, \xi_{c}\right)-K \hat{z}\right) \beta\left(\hat{z}, \xi_{c}\right)^{-1}, \\
\dot{\xi}_{c} & =-\kappa \xi_{c}-C g(y) u, \\
\dot{\hat{z}} & =\left(A_{o}+B_{o} K-G C_{o}\right) \hat{z}+G\left(y+\xi_{c}\right),
\end{aligned}
$$

i) is such that the behavior from the input $v$ in (46) to the states $(z, e)$ is linear and described by the transfer function

$$
\mathcal{W}(s) \triangleq\left[\begin{array}{cc}
s I-A_{z} & B_{o} K+P M \\
0 & s I-A_{e}
\end{array}\right]^{-1}\left[\begin{array}{c}
P \\
P
\end{array}\right],
$$

with $A_{z}=A_{o}+B_{o} K$ and $A_{e}=A_{o}-P M-G C_{o}$;

ii) possesses a spectrally assignable, locally exponentially stable equilibrium point at the origin, with spectrum given by $\left\{\varrho_{\kappa}\right\} \cup \Lambda\left(A_{o}+B_{o} K\right) \cup \Lambda\left(A_{o}-P M-G C_{o}\right)$.

Proof: Consider the system (45) with $\bar{u}=K \hat{z}$. It is straightforward to show that, by definition of the dynamics of $\hat{z}$ as in (47), the error dynamics become

$$
\dot{e}=\left(A_{o}-P M-G C_{o}\right) e+P v .
$$

Finally, by noting that linear terms in $\xi_{c}$ in (45) and in $\xi_{c}$ and $z$ in (49) are canceled out by the structure of the vector field and by rewriting $\bar{u}$ as $\bar{u}=K z-K e$, we obtain that the linearization of the combined system $\left(\xi_{c}, z, e\right)$ is described by

$$
\left[\begin{array}{ccc}
\varrho_{\kappa} & \star & \star \\
0 & A_{o}+B_{o} K & \star \\
0 & 0 & A_{o}-P M-G C_{o}
\end{array}\right]
$$

hence the claim.

Remark 9: It is worth discussing the two main features of the structure of system (41) that have been exploited in the previous construction. First, the definition of the measured output function allows to define a single change of coordinates that transforms the system into a form suitable both for control and observer design. Second, the overall reduced dimension of the dynamic control law - compared to what is expected by considering separately state feedback and observer dynamics - is due to the fact that, despite the observer design needs an extension of dimension equal to that of the system, herein the (available) selection of the control input has been employed to somewhat mimic the effect of the selection of an additional state in the observer dynamics.

\section{B. General Nonlinear Systems}

The extension of the above construction to higherdimensional systems is now presented. To this end, consider 
the change of coordinates

$$
\left[\begin{array}{c}
z \\
\xi
\end{array}\right]=\psi(x, \xi)=\left[\begin{array}{c}
C x+\xi_{1} \\
L_{f}(C x)+\xi_{2} \\
L_{f}^{2}(C x)+\xi_{3} \\
\vdots \\
L_{f}^{n-1}(C x)-\sum_{j=1}^{n-1} \kappa_{j} \xi_{j} \\
\xi
\end{array}\right]
$$

together with the functions $\alpha$ and $\beta$ defined in (19). Definitions similar to the ones considered for planar systems above are also introduced for $\gamma\left(z, \xi_{c}, \bar{u}\right), \delta\left(z, \xi_{c}, \hat{z}, \bar{u}\right)$ and $v=$ $\tilde{\delta}\left(z, \xi_{c}, \hat{z}, \bar{u}\right)-\tilde{\delta}\left(z, \xi_{c}, z, \bar{u}\right)$. For higher-dimensional systems, the following technical assumption is required.

Assumption 6: The functions $L_{g} L_{f}^{i}(C x), i=1, \ldots, n-2$, depends only on $y$.

Finally, note that in the rest of this section the notation $A_{o}$, $B_{o}, C_{o}, P, F$ and $\Gamma$ refers to the matrices introduced in (and around) Proposition 8.

Proposition 10: Consider the system (41) and suppose that there exist constants $\kappa_{j} \in \mathbb{R}, j=1, \ldots, n-1$ such that condition $(C 2)$ of Proposition 4 holds. Let $i) \bar{u}=K \hat{z}$, with $K$ such that $\Lambda\left(A_{o}+B_{o} K\right) \subset \mathbb{C}^{-}$and $\left.i i\right) G$ such that $\Lambda\left(A_{o}-P M-G C_{o}\right) \subset \mathbb{C}^{-}$and suppose that Assumption 6 holds. Then, in the $\xi_{c}, z, e$ coordinates, the system (41) in closed loop with

$$
\begin{aligned}
u & =-\left(\alpha\left(\hat{z}, \xi_{c}\right)-K \hat{z}\right) \beta\left(\hat{z}, \xi_{c}\right)^{-1}, \\
\dot{\xi}_{c, 1} & =\xi_{c, 2}-L_{g}(C x) u, \\
& \vdots \\
\dot{\xi}_{c, n-1} & =-\sum_{j=1}^{n-1} \kappa_{j} \xi_{c, j}-L_{g} L_{f}^{n-2}(C x) u, \\
\dot{\hat{z}} & =\left(A_{o}+B_{o} K-G C_{o}\right) \hat{z}+G\left(y+\xi_{c}\right),
\end{aligned}
$$

i) is such that the behavior from the input $v$ to the states $(z, e)$ is linear and described by the transfer function

$$
\mathcal{W}(s) \triangleq\left[\begin{array}{cc}
s I-A_{z} & B_{o} K+P M \\
0 & s I-A_{e}
\end{array}\right]^{-1}\left[\begin{array}{c}
P \\
P
\end{array}\right]
$$

with $A_{z}=A_{o}+B_{o} K$ and $A_{e}=A_{o}-P M-G C_{o}$;

ii) possesses a spectrally assignable, locally exponentially stable equilibrium point at the origin, with spectrum given by $\Lambda\left(\mathcal{A}_{\kappa}\right) \cup \Lambda\left(A_{o}+B_{o} K\right) \cup \Lambda\left(A_{o}-P M-G C_{o}\right)$.

\section{Numerical Simulations}

The aim of this section is to validate the performances of the proposed schemes by means of numerical simulations.

\section{A. A locally uncontrollable system with non-hyperbolic equilibrium point}

Consider the planar nonlinear system described by

$$
\dot{x}_{1}=x_{1}+x_{2}+u, \quad \dot{x}_{2}=x_{1} x_{2} .
$$

The linearization of system (54) around the origin, described by the matrices

$$
A=\left[\begin{array}{ll}
1 & 1 \\
0 & 0
\end{array}\right], \quad B=\left[\begin{array}{l}
1 \\
0
\end{array}\right],
$$

possesses an uncontrollable mode at zero, hence it is an uncontrollable system with a non-hyperbolic equilibrium point. Letting $y_{\ell}=x_{1}-x_{2}$, namely $H=[1,-1]$, be the desired output, the conditions $(C 1)$ and $(C 2)$ hold. The resulting dynamic control law (5) becomes

$$
\left\{\begin{aligned}
u= & \frac{1}{1-x_{2}+\kappa}\left(-x_{1}-x_{2}+x_{2}^{2}+x_{1}^{2} x_{2}-\kappa^{2} \xi\right. \\
& \left.-c_{1}\left(x_{1}-x_{2}+\xi\right)-c_{2}\left(x_{1}+x_{2}-x_{1} x_{2}-\kappa \xi\right)\right), \\
\dot{\xi}= & -\kappa \xi-u
\end{aligned}\right.
$$

with $c_{1}$ and $c_{2}$ positive constants, and allows to enforce local asymptotic stability of the zero equilibrium point of (54). The

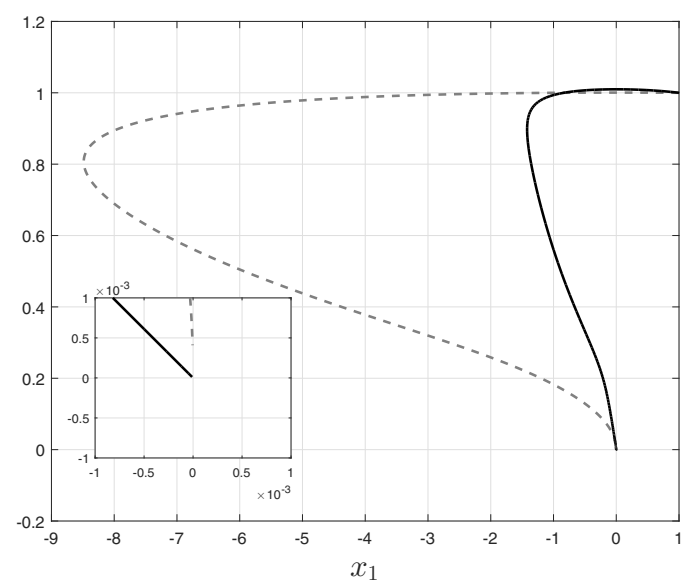

Fig. 8. Trajectories of system (54), initialized at $x(0)=[1,1]$, in closed loop with the dynamic control (56) (solid line) and with the static control law (57) (dashed line).

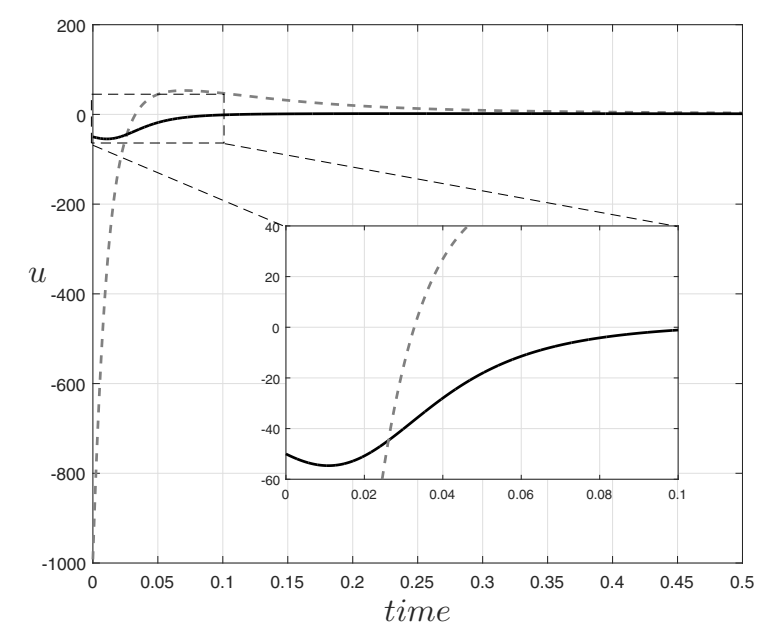

Fig. 9. Time histories of the control $\boldsymbol{u}$ (56) (solid line) and the static control law (57) (dashed line).

dynamic control law (56) is compared with the static control law obtained by enforcing, via the selection of the output, a minimum-phase zero dynamics to system (54). This can be achieved by letting $y_{z}=x_{1}+q_{1} x_{2}^{2}, q_{1}>0$, which is such that $x_{1}=-q_{1} x_{2}^{2}$ on the output-zeroing submanifold, yielding 
an asymptotically stable zero-dynamics described by $\dot{x}_{2}=$ $-q_{1} x_{2}^{3}$. The control law solving the output-zeroing problem is then given by

$$
u_{z}=-x_{1}-x_{2}-2 q_{1} x_{1} x_{2}^{2}-q_{2}\left(x_{1}+q_{1} x_{2}^{2}\right),
$$

with $q_{2}$ a positive constant. The benefit of ensuring exponential convergence of the sum and the difference of $x_{1}$ and $x_{2}$ to an asymptotically converging trajectory of the $\xi$ subsystem, yielded by the control law (56), can be appreciated by the numerical comparison in Figure 8. The figure depicts the trajectories of system (54), initialized at $x(0)=[1,1]$, in closed loop with the dynamic control law (56) (solid line) and with the static control law (57) (dashed line), with the two closed-loop systems simulated for the same amount of time. Figure 9 displays the time histories of the resulting control inputs (56) (solid line) and (57) (dashed line).

\section{B. Output feedback stabilization of a locally uncontrollable system with non-hyperbolic equilibrium point}

Consider the planar nonlinear system described by

$$
\begin{aligned}
\dot{x}_{1} & =x_{1}+x_{2}+u, \quad \dot{x}_{2}=x_{1} x_{2}^{a}, \\
y & =x_{1},
\end{aligned}
$$

with $a \in \mathbb{N}$, which possesses, similarly to the previous example, a linearly uncontrollable non-hyperbolic equilibrium point. It can be checked that Assumption 2 holds, hence the transformation $\psi\left(x, \xi_{c}\right)$ defined in (42) qualifies as a welldefined change of coordinates, described in particular by $z_{1}=$ $x_{1}+\xi_{c}, z_{2}=x_{1}+x_{2}-\kappa \xi_{c}$. The state feedback control law for system (58) is given by $\dot{\xi}_{c}=-\kappa \xi_{c}-u=\sigma\left(x, \xi_{c}\right)$, $u=(\kappa+1)^{-1}\left(-x_{1}-x_{2}-x_{1} x_{2}^{a}-\kappa^{2} \xi_{c}+v\right)$. Interestingly, the equilibrium $\xi_{c}=0$ of $\dot{\xi}_{c}=\sigma\left(0, \xi_{c}\right)$ is not asymptotically (locally exponentially) stable in the first approximation, yet it can be shown that $\sigma\left(0, \xi_{c}\right)=-(\kappa+1)^{a-1} \xi_{c}^{a+1}$, hence it is locally asymptotically stable for any $\kappa>0$ and for $a=2 m$, with $m \in \mathbb{N}$. The output feedback control law is described by

$$
\begin{aligned}
u & =-(\kappa+1)^{-1}\left(-\hat{x}_{1}-\hat{x}_{2}-\hat{x}_{1} \hat{x}_{2}^{a}-\kappa^{2} \xi_{c}+K \hat{z}\right), \\
\dot{\xi}_{c} & =-\kappa \xi_{c}-u, \dot{\hat{z}}=\left(A_{o}+B_{o} K-G C_{o}\right) \hat{z}+G\left(y+\xi_{c}\right)
\end{aligned}
$$

with $\hat{x}_{1}=\hat{z}_{1}-\xi_{c}$ and $\hat{x}_{2}=\hat{z}_{2}-\hat{z}_{1}+(\kappa+1) \xi_{c}$. In the following numerical simulations, with $a=2$, the matrices $K$ and $G$ have been selected such that $\Lambda\left(A_{o}+B_{o} K\right)=\{-1,-1\}$ and $\Lambda\left(A_{o}-P M-G C_{o}\right)=\{-10,-11\}$, respectively. Figure 10 depicts the trajectories of the system (58) in closed loop with the state feedback control law defined above and the output feedback (59), gray and black lines, respectively, initialized at $x(0)=[0,1]^{\top}$ and the remaining components of the dynamic control laws initially at zero.

\section{The Ball and Beam}

Consider the Ball and Beam model described by [11]

$$
\begin{aligned}
\dot{r} & =v, \quad \dot{v}=\bar{b}\left(r \omega^{2}-\bar{g} \sin (\phi)\right), \\
\dot{\phi} & =\omega, \quad \dot{\omega}=u,
\end{aligned}
$$

with $r(t) \in \mathbb{R}$ and $v(t) \in \mathbb{R}$ denoting the position and the velocity of the ball on the beam, respectively, while $\phi(t) \in \mathbb{R}$

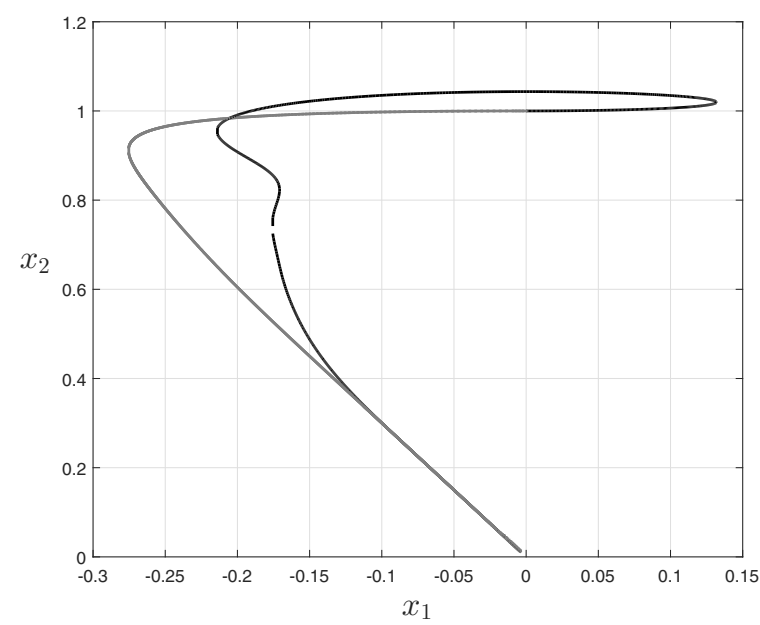

Fig. 10. Trajectories of system (58), initialized at $\boldsymbol{x}(\mathbf{0})=[\mathbf{0 , 1}]^{\top}$, in closed loop with the state feedback control law (gray line) and the output feedback law (59) (black line).

and $\omega(t) \in \mathbb{R}$ denote the angle and the angular velocity, respectively, of the beam with respect to the horizontal line. The constant $\bar{g}$ represents gravity, while $\bar{b}=m /\left(J_{b} / R^{2}+m\right)$, where $m$ denotes the mass, $J_{b}$ the moment of inertia and $R$ the radius of the ball. The numerical values are taken from [11]: $\bar{b}=0.7143$ and, clearly, $\bar{g}=9.81 \mathrm{~m} / \mathrm{s}^{2}$. Let, for simplicity $x=[r, v, \phi, \omega]^{\top}$. The nonlinear terms in the second equation are due to the inclination of the beam and the centrifugal acceleration (Corolis term), respectively. It can be shown that system (60) is not feedback linearizable, namely there does not exist any output function such that the system has relative degree four [25]. The objective here is then to steer the output $y=x_{1}$, i.e. the position of the ball, to asymptotically track a desired reference signal $y_{d}$ [11]. It can be easily shown that the relative degree of the ball and beam model with the considered output is not well-defined, hence the exact inputoutput linearization approach is not applicable to solve the tracking problem. Recall also that the system is not feedback linearizable at all [11]. The choice $H=[1,0,0,0]$ satisfies the condition $\left(C 1^{\prime}\right)$ of Proposition 5, namely the output $y_{\ell}=x_{1}$ is such that the linearized system is observable. It is straightforward to notice that $H g(x)=H \nabla_{x} f(x) g(x)=0$. The change of coordinates is obtained by letting

$$
\begin{aligned}
z_{i} & =x_{i}+\xi_{i}, \quad i=1,2 \\
z_{3} & =-\bar{b} \bar{g} \sin \left(x_{3}\right)+x_{1} x_{4}^{2}+\xi_{3}, \\
z_{4} & =\bar{b}\left(x_{2} x_{4}^{2}-\bar{g} \cos \left(x_{3}\right) x_{4}\right)-\sum_{j=1}^{3} \kappa_{j} \xi_{j}
\end{aligned}
$$

which yields a transformation locally defined around the origin. Therefore, the dynamic control law (19) becomes

$$
\left\{\begin{aligned}
u_{b}= & \frac{1}{2 x_{2} x_{4}-\bar{g} \cos \left(x_{3}\right)+2 \kappa_{3} x_{1} x_{4}}\left(-x_{4}^{2}\left(-\bar{g} \sin \left(x_{3}\right)\right.\right. \\
& \left.+x_{1} x_{4}^{2}\right)-\bar{g} \sin \left(x_{3}\right) x_{4}^{2}+\kappa_{1} \xi_{2}+\kappa_{2} \xi_{3}-\kappa_{1} \kappa_{3} \xi_{1} \\
& \left.-\kappa_{2} \kappa_{3} \xi_{2}-\kappa_{3}^{2} \xi_{3}+v\right) \\
\dot{\xi}_{i}= & \xi_{i+1}, \quad i=1,2 \\
\dot{\xi}_{3}= & -\kappa_{1} \xi_{1}-\kappa_{2} \xi_{2}-\kappa_{3} \xi_{3}-2 x_{1} x_{4} u_{b} .
\end{aligned}\right.
$$



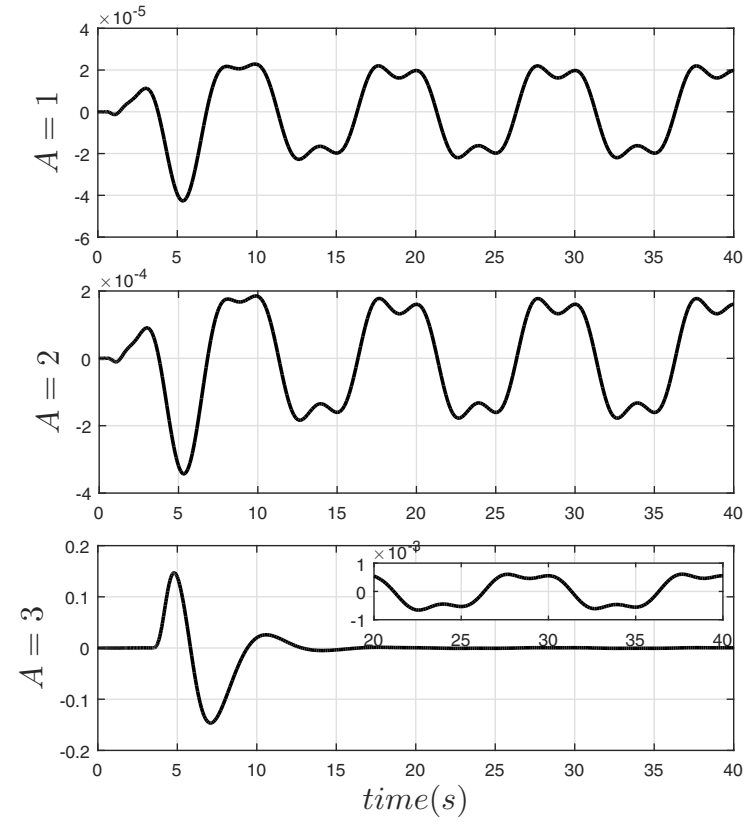

Fig. 11. Time histories of the first component of the dynamic extension $\xi(t)$, corresponding to $A=1, A=2$ and $A=3$, top, middle and bottom graph, respectively, which provide the steady-state approximation bound discussed in Proposition 6.

By selecting $v=-\sum_{i=1}^{4} c_{i}\left(z_{i}-y_{d}^{(i-1)}\right)+y_{d}^{(4)}$, with $c_{i}$, $i=1, \ldots, 4$ such that the polynomial $c_{1}+c_{2} s+c_{3} s^{2}+c_{4} s^{3}+s^{4}$ is Hurwitz, and by letting $\kappa_{i}, i=1,2,3$ be such that the zero equilibrium of the $\xi$ dynamics, with $z=0$, is locally exponentially stable, the dynamic control law (62) ensures local asymptotic stability of the zero equilibrium point of the ball and beam model (60), with $y_{d}(t) \equiv 0$, and approximate tracking of $y_{d}$. Similar numerical simulations are performed in [11] in the case $y_{d}(t)=A \cos (\pi t / 5)$, in which two alternative approximation techniques are proposed and compared with the simple linearization method (Jacobian approximation). It is shown in particular that the latter scheme does not guarantee boundedness of the trajectories for $A=6$, while the best of the two approximations provides the following approximation errors $^{3}: 1.3 \cdot 10^{-4}$ for $A=1 ; 1.0 \cdot 10^{-3}$ for $A=2 ; 3.6 \cdot 10^{-3}$ for $A=3$ and $3.3 \cdot 10^{-2}$ for $A=6$. The graphs in Figure 11 depict the time histories of the first component of the dynamic extension $\xi(t)$, corresponding to $A=1, A=2$ and $A=3$, top, middle and bottom graph, respectively, showing a more accurate tracking with respect to the results of [11]. The comparison between the tracking errors yielded by the control law proposed in [11] and (62) is summarized in Table 1.

Table 1. Comparison with [11]

\begin{tabular}{|c||c|c|c|c|}
\hline & $A=1$ & $A=2$ & $A=3$ & $A=6$ \\
\hline \hline$[11]$ & $1.3 \cdot 10^{-4}$ & $1 \cdot 10^{-3}$ & $3.6 \cdot 10^{-3}$ & $3.3 \cdot 10^{-2}$ \\
\hline$(62)$ & $6 \cdot 10^{-5}$ & $5 \cdot 10^{-4}$ & $1.5 \cdot 10^{-3}$ & $9 \cdot 10^{-3}$ \\
\hline
\end{tabular}

Finally, concerning the case $A=6$, Figure 12 shows the time histories of the position and velocity of the ball and the

\footnotetext{
${ }^{3}$ The errors are computed by considering the infinity norm of the distance between the actual output and the reference signal between $30 \mathrm{~s}$ and $40 \mathrm{~s}$.
}
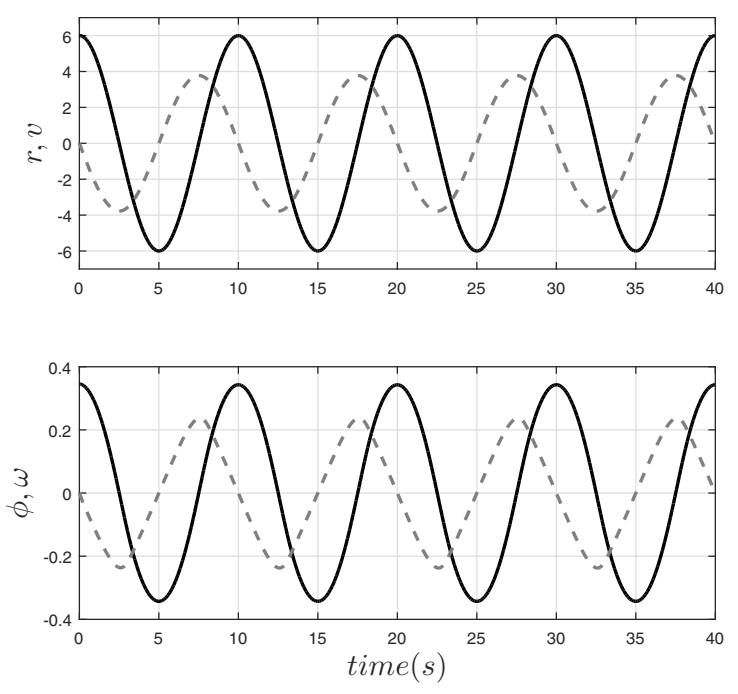

Fig. 12. Top graph: time histories of the position $r(t)$ and the velocity $\boldsymbol{v}(t)$ of the ball with system $(60)$ initialized at $x(0)=[0.5,0,0,0]^{\top}$ in closed-loop with (62), black and gray lines, respectively. Bottom graph: time histories of the angular position $\phi(t)$ and velocity $\omega(t)$ of the beam with system (60) in closed-loop with (62), black and gray lines, respectively.

angle and angular velocity of the beam, top and bottom graph, respectively, initialized at $x(0)=[6,0,0.3450]^{\top}$, while the time histories of the state of the dynamic extension $\xi(t)$ initialized at $\xi(0)=[0,0,0]^{\top}$ are reported in Figure 13.

\section{CONCLUSIONS}

In this paper approximate feedback linearization has been revisited by introducing a dynamic extension, which allows, under mild assumptions, to immerse a nonlinear system with state of dimension $n$ into a chain of $n$ integrators, hence with linear input/output behavior, which "contains" all the components of the underlying nonlinear system. This result is achieved systematically and without resorting to the solution of any partial differential equation, also for nonlinear systems that are not linearly controllable, hence feedback linearizable in the classical sense. The construction is then specialized to provide a linear design technique to define control laws that enforce (local) asymptotic stability of an equilibrium point or local asymptotic tracking of reference signals. Similar ideas have been then applied to the problem of designing observers for nonlinear systems with linear error dynamics: the resulting construction consists of a change of coordinates that is based on a combination of repeated output derivatives and (implicit) integrals. The two techniques are combined to define an output feedback control law for nonlinear systems, thus establishing a nonlinear separation principle. Note that the architecture consisting of a nonlinear inner loop allows use of linear tools in the design stage. On-going effort is devoted to extend the proposed results - which in this paper mainly exhibit a local nature around a certain equilibrium point of the underlying nonlinear systems - to hold globally in the entire state-space. 

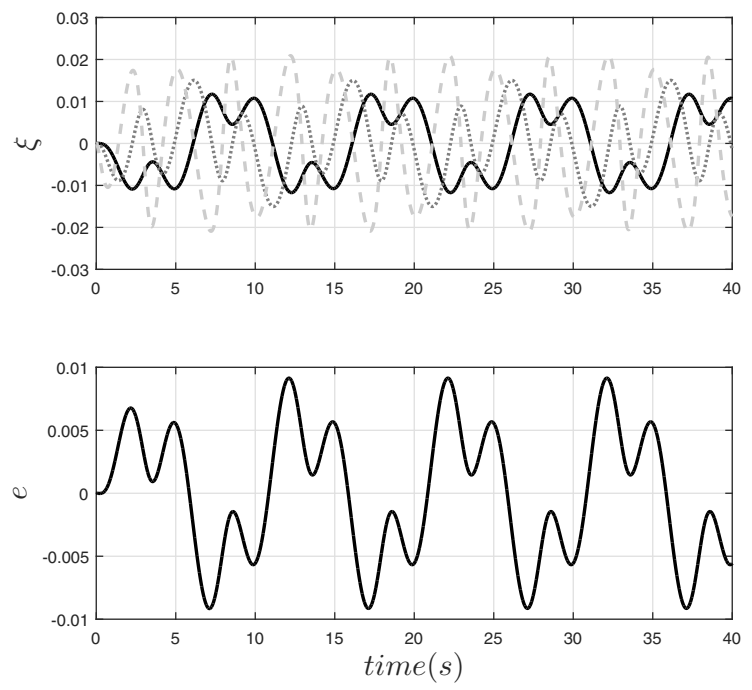

Fig. 13. Time histories of the state of the dynamic extension $\boldsymbol{\xi}(\boldsymbol{t})$ initialized at $\xi(0)=[\mathbf{0}, \mathbf{0}, \mathbf{0}]^{\top}$ (top graph) and of the tracking error $e(t)$ (bottom graph).

\section{REFERENCES}

[1] V. Andrieu and L. Praly. On the existence of a Kazantzis/Kravaris/ Luenberger observer. SIAM Journal on Control and Optimization, 45:432-456, 2013.

[2] D. Astolfi and L. Marconi. A high-gain nonlinear observer with limited gain power. IEEE Transactions on Automatic Control, 60(11):30593064,2015

[3] A. Banaszuk and J. Hauser. Approximate feedback linearization: A homotopy operator approach. SIAM Journal of Control and Optimization, 34(5):1533-1554, 1996.

[4] A. Banaszuk, A. Swiech, and J. Hauser. Approximate feedback linearization: Least squares approximate integrating factors. In Proc. of the 33rd Conference on Decision and Control Lake Buena Vista, FL, pages 1621-1626, 1994.

[5] S.A. Bortoff. Approximate state-feedback linearization using spline functions. Automatica, 33(8):1449-1458, 1997.

[6] F. Esfandiari and H.K. Khalil. Output feedback stabilization of fully linearizable systems. International Journal of control, 56(5):1007-1037, 1992.

[7] G. O. Guardabassi and S. M. Savaresi. Foundations of feedback theory for nonlinear dynamical systems. IEEE Transactions on Circuit and Systems, 27(2):104-123, 1980.

[8] G. O. Guardabassi and S. M. Savaresi. Approximate feedback linearization of discrete-time nonlinear systems using virtual input direct design. Systems and Control Letters, 32:63-74, 1997.

[9] G.O. Guardabassi and S.M. Savaresi. Approximate linearization via feedback - an overview. Automatica, 37(1):1-15, 2001.

[10] J. Hauser. Nonlinear control via uniform system approximation. Systems and Control Letters, 17:145-154, 1991.

[11] J. Hauser, S. Sastry, and P. Kokotovic. Nonlinear control via approximate input-output linearization: The Ball and Beam example. IEEE Transactions on Automatic Control, 37(3):392-398, 1992.

[12] A. Isidori. The matching of a prescribed linear input-output behavior in a nonlinear system. IEEE Transactions on Automatic Control, 30(3):258265, 1985.

[13] A. Isidori. Nonlinear Control Systems. Springer-Verlag, Berlin, 3rd edition, 1995.

[14] A. Isidori and A. Astolfi. Disturbance attenuation and $H_{\infty}$-control via measurement feedback in nonlinear systems. IEEE Transactions on Automatic Control, 37(9):1283-1293, 1992.

[15] A. Isidori and A.J. Krener. On feedback equivalence of nonlinear systems. Systems and Control Letters, 2(2):118-121, 1982.

[16] A. Isidori and A. Ruberti. On the synthesis of linear intput-output response for nonlinear systems. Systems and Control Letters, 4(1):1722,1984 .
[17] Z.-P. Jiang and I. Kanellakopoulos. Global output-feedback tracking for a benchmark nonlinear system. IEEE Transactions on Automatic Control, 45(5):1023-1027, 2000.

[18] T. Kailath. Linear Systems. Prentice Hall, Englewood Cliffs, 1980.

[19] N. Kazantzis and C. Kravaris. Nonlinear observer design using Lyapunov's auxiliary theorem. Systems and Control Letters, 34:241-247, 1998.

[20] H.K. Khalil. Adaptive output feedback control of nonlinear systems represented by input-output models. IEEE Transactions on Automatic Control, 41(2):177-188, 1996.

[21] H.K. Khalil. High-gain observers in nonlinear feedback control. In $H$. Nijmeijer and T.I. Fossen, editors, New Directions in Nonlinear Observer Design, volume 244 of Lecture Notes in Control and Information Sciences, pages 249-268, 1999.

[22] H.K. Khalil and L. Praly. High-gain observers in nonlinear feedback control. International Journal of Robust and Nonlinear Control, 24:9931015, 2014.

[23] A. J. Krener. Approximate linearization by state feedback and coordinate change. Systems and Control Letters, 5:181-185, 1984.

[24] D.G. Luenberger. Observing the state of a linear system. IEEE Transactions on Military Electronics, 8:74-80, 1964.

[25] R. Marino. On the largest feedback linearizable subsystem. Systems and Control Letters, 6:345-351, 1986.

[26] L. Praly and A. Astolfi. Global asymptotic stabilization by output feedback under a state norm detectability assumption. In Proc. of the 44th IEEE Conference on Decision and Control, pages 2634-2639, 2005.

[27] L Praly and Z.-P. Jiang. Stabilization by output feedback for systems with ISS inverse dynamics. Systems \& Control Letters, 21(1):19-33, 1993.

[28] L. Praly and Z.-P. Jiang. Linear output feedback with dynamic high gain for nonlinear systems. Systems and Control Letters, 53(2):107116, 2004.

[29] M. Sassano and A. Astolfi. Approximate dynamic tracking and feedback linearization. In Proc. of the 55th IEEE Conference on Decision and Control, Las Vegas, 2016.

[30] A. J. Stack and F. J. Doyle III. Application of a controllaw nonlinearity measure to the chemical reactor analysis. Process Systems Engineering, 43(2):425-439, 1997.

[31] A. Teel and L. Praly. Global stabilizability and observability imply semiglobal stabilizability by output feedback. Systems \& Control Letters, 22(5):313-325, 1994.

[32] A. Teel and L. Praly. Tools for semiglobal stabilization by partial state and output feedback. SIAM Journal on Control and Optimization, 33(5):1443-1488, 1995

[33] A. Tornambe. High-gain observers for nonlinear systems. International Journal of Systems Science, 23(9):1475-1489, 1992.

[34] A. Tornambe. Output feedback stabilization of a class of non-minimum phase nonlinear systems. Systems and Control Letters, 19:193-204, 1992.

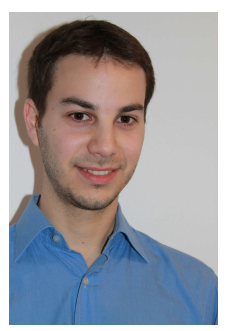

Mario Sassano was born in Rome, Italy, in 1985. He received the B.S degree in Automation Systems Engineering and the M.S degree in Systems and Control Engineering from the University of Rome "La Sapienza", Italy, in 2006 and 2008, respectively. In 2012 he was awarded a Ph.D. degree by Imperial College London, UK, where he had been a Research Assistant in the Department of Electrical and Electronic Engineering since 2009. Currently he is an Assistant Professor at the University of Rome "Tor Vergata", Italy. His research interests are focused on nonlinear observer design, optimal control and differential game theory with applications to mechatronical systems and output regulation for hybrid systems. He is Associate Editor of the IEEE CSS Conference Editorial Board and of the EUCA Conference Editorial Board. 


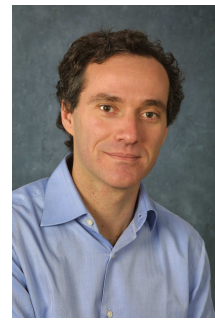

Alessandro Astolfi was born in Rome, Italy, in 1967. He graduated in Electrical Engineering from the University of Rome "La Sapienza" in 1991. In 1992 he joined ETH-Zurich where he obtained a M.Sc. in Information Theory in 1995 and the Ph.D. degree with Medal of Honor in 1995 with a Thesis on Discontinuous Stabilization of Nonholonomic Systems. In 1996 he was awarded a Ph.D. from the University of Rome "La Sapienza" for his work on nonlinear robust control. Since 1996 he has been with the Electrical and Electronic Engineering Department of Imperial College London, London (UK), where he is currently Professor of Nonlinear Control Theory and Head of the Control and Power Group (2010-). From 1998 to 2003 he was also an Associate Professor at the Dept. of Electronics and Information of the Politecnico of Milano. Since 2005 he has also been a Professor at Dipartimento di Ingegneria Civile e Ingegneria Informatica, University of Rome "Tor Vergata". He has been a visiting lecturer in "Nonlinear Control" in several universities, including ETH-Zurich (1995-1996); Terza University of Rome (1996); Rice University, Houston (1999); Kepler University, Linz (2000); SUPELEC, Paris (2001).

His research interests are focused on mathematical control theory and control applications, with special emphasis for the problems of discontinuous stabilization, robust and adaptive control, observer design and model reduction. He is the author of more than 120 journal papers, of 30 book chapters and of over 240 papers in refereed conference proceedings. He is the recipient of the IEEE CSS A. Ruberti Young Researcher Prize (2007), of the IEEE CSS George S. Axelby Outstanding Paper Award (2012) and the IFAC Best Paper Award (2017). He is a "Distinguished Member" of the IEEE CSS. He is the author (with D. Karagiannis and R. Ortega) of the monograph "Nonlinear and Adaptive Control with Applications" (Springer-Verlag).

$\mathrm{He}$ is Associate Editor of the International Journal of Control and Area Editor of the International Journal of Adaptive Control and Signal Processing. He is Senior Editor of the IEEE Trans. on Automatic Control and Editor-in-Chief of the European Journal of Control. He has also served in the IPC of various international conferences. He is currently the Chair of the IEEE CSS Conference Editorial Board (2010-). 